\title{
Article \\ Wormhole Solutions in Symmetric Teleparallel Gravity with Noncommutative Geometry
}

\author{
Zinnat Hassan ${ }^{1,+}+\mathbb{D}$, Ghulam Mustafa ${ }^{2,+}+\mathbb{C}$ and Pradyumn Kumar Sahoo ${ }^{1, *,+(\mathbb{C})}$ \\ 1 Department of Mathematics, Birla Institute of Technology and Science-Pilani, Hyderabad Campus, \\ Hyderabad 500078, India; p20190463@hyderabad.bits-pilani.ac.in \\ 2 Department of Mathematics, Shanghai University, Shanghai 200444, China; mustafa123@shu.edu.cn \\ * Correspondence: pksahoo@hyderabad.bits-pilani.ac.in; Tel.: +91-9912564154 \\ + These authors contributed equally to this work.
}

check for updates

Citation: Hassan, Z.; Mustafa, G.; Sahoo, P.K. Wormhole Solutions in Symmetric Teleparallel Gravity with Noncommutative Geometry. Symmetry 2021, 13, 1260. https:// doi.org/10.3390/sym13071260

Academic Editors: Daniele Pranzetti and Kazuharu Bamba

Received: 13 April 2021

Accepted: 1 July 2021

Published: 14 July 2021

Publisher's Note: MDPI stays neutral with regard to jurisdictional claims in published maps and institutional affiliations.

Copyright: (c) 2021 by the authors. Licensee MDPI, Basel, Switzerland. This article is an open access article distributed under the terms and conditions of the Creative Commons Attribution (CC BY) license (https:/ / creativecommons.org/licenses/by/ $4.0 /)$.

\begin{abstract}
This article describes the study of wormhole solutions in $f(Q)$ gravity with noncommutative geometry. Here, we considered two different $f(Q)$ models-a linear model $f(Q)=\alpha Q$ and an exponential model $f(Q)=Q-\alpha\left(1-e^{-Q}\right)$, where $Q$ is the non-metricity and $\alpha$ is the model parameter. In addition, we discussed the existence of wormhole solutions with the help of the Gaussian and Lorentzian distributions of these linear and exponential models. We investigated the feasible solutions and graphically analyzed the different properties of these models by taking appropriate values for the parameter. Moreover, we used the Tolman-Oppenheimer-Volkov (TOV) equation to check the stability of the wormhole solutions that we obtained. Hence, we found that the wormhole solutions obtained with our models are physically capable and stable.
\end{abstract}

Keywords: wormholes; non-commutative; stability; $f(Q)$ gravity

\section{Introduction}

Einstein's general theory of relativity permits the use of traversable wormholes to describe topological passage through a hypothetical bridge joining two different universes or two different parts of the universe. The concept of the wormhole was first proposed by Flamm [1]. He built up the Schwarzschild solution of isometric embedding. Wheeler and Misner [2] first introduced the term "wormhole" in 1957. Morris and Thorne [3] presented a spherically symmetric metric that associates areas of asymptotic flatness. There are some types of inhomogeneous matter that are the fundamental elements for the construction of wormholes, and this matter disagrees with the energy conditions, whereas normal matter fulfills the energy conditions. Matter that violates the energy conditions, especially the null energy condition (NEC), is called exotic matter.

Exotic matter is a hypothetical type of matter that is needed to violate the null energy condition (NEC), and it is the fundamental ingredient for forming a traversable wormhole. With the assistance of the event horizon, in 1935, Einstein and Rosen [4] proposed wormhole solutions that are known as Lorentzian wormholes or Schwarzschild wormholes. Generally, classical matter satisfies the energy conditions, but the Casimir effect and the Hawking evaporation are some quantum fields that violate the energy conditions. Because of its arguable nature, the limit of the utilization of exotic matter is a significant issue. The modified theories of gravity $[5,6]$ may avoid the violation with the actual matter source, and the effective energy-momentum tensor is responsible for the violation of the NEC and the presence of ordinary matter strings in the wormhole in order to fulfill the energy conditions.

Lobo and Oliveira [7] discussed the wormhole solution by using a specific shape function in the $f(R)$ theory of gravity and investigated the legitimacy of the energy conditions. Azizi [8] examined the wormhole solutions in $f(R, T)$ gravity, where $R$ and $T$ denote the scalar curvature and the trace of the stress-energy tensor, respectively. Azizi 
expected that in $f(R, T)$ gravity, the matter threading the wormhole might fulfill the energy conditions; hence, for the violation of the null energy condition, the effective stress-energy is responsible. By utilizing anisotropic matter, DeBenedictis and Horvat [9] found the throat radii for the $R^{n}$ model. Garcia and Lobo [10] considered the Brans-Dicke theory to study the exact wormhole solutions. Böhmer et al. [6] derived traversable wormhole solutions for a particular $f(T)$ model with redshift and a shape function, and they showed that wormholes that obey the energy conditions exist. Moreover, Jamil and his collaborator [11] derived exact static wormhole solutions for isotropic, anisotropic, and barotropic pressure for a linear equation of state (EoS), and it was found that the energy conditions were obeyed in the isotropic and barotropic cases, while in the anisotropic cases, it was violated in a modified $f(T)$ theory of gravity. Jimenez et al. [12] proposed symmetric teleparallel gravity, i.e., $f(Q)$ gravity where the non-metricity $Q$ drives the gravitational interaction. Symmetric teleparallel gravity is the curvature-free and torsion-free theory of gravitation. The investigations on $f(Q)$ gravity have grown quickly in both the theoretical and observational fields (see References [13-19]). Recently, Hassan et al. [20] studied traversable wormhole geometries in $f(Q)$ gravity. They assumed the relation between radial and lateral pressure, a phantom energy equation of state, and some particular cases of specific shape functions, and they discussed their properties by using the energy conditions.

As stated in [21], the concept of noncommutative geometry is an intrinsic property of the manifold itself, rather than a superimposed geometrical structure, so the idea of noncommutativity can be used in modified theories of gravity in addition to GR. It is interesting to mention here that Mathew and Andrew [22] provided the background of both noncommutative distributions. On the other hand, the actual presence of the Planck length requires the mathematical background for high-energy physics to be improved. It follows that conventional geometrical concepts and notions may not be well suited to explaining the physical phenomena at minimal distances. Furthermore, some drastic changes in physics are expected close to the Planck scale, with one imperative and interesting consequence being the appearance of the noncommutative structure of spacetime. The basic idea behind noncommutativity in spacetime is very much inspired by quantum mechanics. Spacetime quantization is the crucial improvement of string theory, and the coordinates of spacetime may be treated as noncommutative operators in the D-brane [23]. Such operators are used in a basic discretization of spacetime because of the commutator $\left[x^{\mu}, x^{\nu}\right]=i \Theta^{\mu \nu}$, where $\Theta^{\mu \nu}$ is the antisymmetric matrix that indicates the discretization of spacetime. Noncommutativity is utilized to supplant the point-like structures with smeared objects in order to wipe out the divergences that show up in general relativity. This smearing can be displayed by the utilization of Gaussian and Lorentzian distributions of minimal length $\sqrt{\Theta}$, rather than the Dirac delta function. Along these lines, the energy densities of the particle-like, stationary, spherically symmetric gravitational source take the accompanying structures [21,24,25]:

$$
\rho=\frac{M e^{-\frac{r^{2}}{4 \Theta}}}{8 \pi^{3 / 2} \Theta^{3 / 2}}, \quad \rho=\frac{\sqrt{\Theta} M}{\pi^{2}\left(\Theta+r^{2}\right)^{2}},
$$

respectively. Here, $M$ is the total mass, and it can be a centralized object diffused over the area of a linear dimension $\sqrt{\Theta}$, similarly to a wormhole [26]. These energy densities embed the quantum effects of noncommutative spacetime. These effects will be as strong as $r \approx \sqrt{\Theta}$. In this region, we expect the emergence of new physical phenomena due to the noncommutativity. Thus, this approach lies in a semi-classical perspective rather than in a quantum theory for the gravitational field. Sushkov [27] also used a Gaussian source to demonstrate phantom-energy-supported wormholes, just as Nicolini and Spalluci did [28], in order to display the physical effects of short-distance changes in noncommutative directions in the investigation of black holes. Rahaman and his collaborator [26] explored wormhole solutions in a noncommutative background and derived their reality in four and five dimensions as well. Abreu and Sasaki [29] found that the wormhole solutions in a noncommutative background fulfilled the energy conditions in the absence of exotic 
matter. Jamil et al. [30] also investigated the wormhole solutions in $f(R)$ gravity in the same background.

In this article, we studied a spherically symmetric, static wormhole in symmetric teleparallel gravity with noncommutative geometry. This article is organized as follows. Section 2 contains the basic formulation for $f(Q)$ gravity. We introduce the fundamental conditions for traversable wormholes in Section 3. We thoroughly discuss wormhole geometries in $f(Q)$ gravity and the energy conditions in the same section. In Section 4, we study the wormhole solutions for the Gaussian and Lorentzian distributions using linear and exponential gravity models. Section 5 provides a discussion of the stability analysis for both wormhole solutions. In addition, an anisotropy analysis for Gaussian and Lorentzian sources for both gravity models is discussed in Section 6. Finally, the conclusions and discussions of this manuscript are highlighted in Section 7.

\section{Basic Field Equations in $f(Q)$ Gravity}

Consider the proposal of symmetric teleparallel gravity, i.e., $f(Q)$ gravity given by the action [12]

$$
\mathcal{S}=\int \frac{1}{2} f(Q) \sqrt{-g} d^{4} x+\int \mathcal{L}_{m} \sqrt{-g} d^{4} x
$$

where $\mathcal{L}_{m}$ represents the matter's Lagrangian density, $g$ is the determinant of the metric $g_{\mu v}$, and $f(Q)$ represents an arbitrary function of $Q$.

The non-metricity tensor is defined as

$$
Q_{\lambda \mu v}=\nabla \lambda g_{\mu v}
$$

and its trace can be written as

$$
Q_{\alpha}=Q_{\alpha}{ }_{\mu}^{\mu}, \tilde{Q}_{\alpha}=Q^{\mu}{ }_{\alpha \mu} .
$$

In addition, it is helpful to write the superpotential in terms of non-metricity:

$$
P^{\alpha}{ }_{\mu \nu}=\frac{1}{4}\left[-Q^{\alpha}{ }_{\mu \nu}+2 Q_{(\mu}{ }^{\alpha}{ }_{\nu)}+Q^{\alpha} g_{\mu v}-\tilde{Q}^{\alpha} g_{\mu \nu}-\delta_{(\mu}^{\alpha} Q_{v)}\right]
$$

One can readily check that the non-metricity can also be written in the form [12]:

$$
Q=-Q_{\alpha \mu \nu} P^{\alpha \mu \nu}
$$

Again, the energy-momentum can be written as

$$
T_{\mu \nu}=-\frac{2}{\sqrt{-g}} \frac{\delta\left(\sqrt{-g} \mathcal{L}_{m}\right)}{\delta g^{\mu \nu}} .
$$

Now, by varying the action (2) with respect to metric tensor $g_{\mu v}$, one can get the following field equation:

$$
\frac{2}{\sqrt{-g}} \nabla_{\gamma}\left(\sqrt{-g} f_{Q} P^{\gamma}{ }_{\mu \nu}\right)+\frac{1}{2} g_{\mu \nu} f+f_{Q}\left(P_{\mu \gamma i} Q_{\nu}{ }^{i}-2 Q_{\gamma i \mu} P^{\gamma i}{ }_{\nu}\right)=-T_{\mu \nu},
$$

where $f_{Q}=\frac{d f}{d Q}$.

In addition, by varying (2) with respect to the connection, one can get

$$
\nabla \mu \nabla v\left(\sqrt{-g} f_{Q} P_{\mu \nu}^{\gamma}\right)=0 .
$$




\section{Basic Conditions for Traversable Wormholes}

We consider that a spherically symmetric static spacetime for wormhole geometry is defined as

$$
d s^{2}=-e^{2 \Phi(r)} d t^{2}+\left(1-\frac{b(r)}{r}\right)^{-1} d r^{2}+r^{2} d \theta^{2}+r^{2} \sin ^{2} \theta d \phi^{2}
$$

where $b(r)$ and $\Phi(r)$ denote the shape function and redshift function, respectively, and both are functions of the radial coordinate $r$. The radial coordinate $r$ lies in the range $r_{0} \leq r<\infty$, where $r_{0}$ refers to the radius of the throat. To avoid the presence of event horizons, the redshift function $\Phi(r)$ must be finite everywhere. To have a traversable wormhole geometry, $b(r)$ must obey the following conditions:

- Throat condition: $b\left(r_{0}\right)=r_{0}$ and $b(r)$ should be less than $r$ for $r>r_{0}$.

- Flare-out condition: $b^{\prime}\left(r_{0}\right)<1$, i.e., $\frac{b(r)-r b^{\prime}(r)}{b^{2}(r)}>0$, where' represents the derivative with respect to $r$.

- Asymptotic flatness condition: $\frac{b(r)}{r} \rightarrow o$ as $r \rightarrow \infty$.

In addition, the proper radial distance $l(r)$ is defined as

$$
\ell(r)= \pm \int_{r_{0}}^{r} \frac{d r}{\sqrt{1-\frac{b(r)}{r}}} .
$$

$\ell(r)$ must be finite everywhere over $r>r_{0}$, and the expression \pm addresses the two opposite symmetric parts that are associated through the wormhole structure.

In order to proceed further, we presume that the matter content is an anisotropic stress-energy tensor, which can be written as

$$
T_{\mu}^{v}=\left(\rho+P_{t}\right) u_{\mu} u^{v}-P_{t} \delta_{\mu}^{v}+\left(P_{r}-P_{t}\right) v_{\mu} v^{v}
$$

where $\rho$ is the energy density. The expression $v_{\mu}$ is a unitary space-like vector in the radial direction and $u_{\mu}$ is a four-velocity vector; they are defined as $u^{\mu}=e^{-2 \phi(r)} \delta_{0}^{\mu}$ and $v^{\mu}=\sqrt{1-\frac{b}{r}} \delta_{1}^{\mu}$, which meet the conditions $v_{\mu} v^{\mu}=-u_{\mu} u^{\mu}=1$. $P_{r}$ and $P_{t}$ represent the radial pressure in the direction of $u_{\mu}$ and the tangential pressure orthogonal to $v_{\mu}$; both are functions of the radial coordinate $r$.

\subsection{Wormhole Geometries in $f(Q)$ Gravity}

In this subsection, we shall discuss the framework of $f(Q)$ gravity with a spherically symmetric static spacetime. For (10), the trace of the non-metricity tensor $Q$ can be written as

$$
Q=-\frac{2}{r}\left(1-\frac{b(r)}{r}\right)\left(2 \Phi^{\prime}(r)+\frac{1}{r}\right) .
$$

Now, using (10) and (12) in (8), we found the following expressions:

$$
\begin{gathered}
{\left[\frac{1}{r}\left(-\frac{1}{r}+\frac{r b^{\prime}(r)+b(r)}{r^{2}}-2 \Phi^{\prime}(r)\left(1-\frac{b(r)}{r}\right)\right)\right] f_{Q}-\frac{2}{r}\left(1-\frac{b(r)}{r}\right) \dot{f}_{Q}-\frac{f}{2}=-\rho,} \\
{\left[\frac{2}{r}\left(1-\frac{b(r)}{r}\right)\left(2 \Phi^{\prime}(r)+\frac{1}{r}\right)-\frac{1}{r^{2}}\right] f_{Q}+\frac{f}{2}=-P_{r,}} \\
{\left[\frac{1}{r}\left(\left(1-\frac{b(r)}{r}\right)\left(\frac{1}{r}+\Phi^{\prime}(r)\left(3+r \Phi^{\prime}(r)\right)+r \Phi^{\prime \prime}(r)\right)-\frac{r b^{\prime}(r)-b(r)}{2 r^{2}}\left(1+r \Phi^{\prime}(r)\right)\right)\right] f_{Q}+} \\
\frac{1}{r}\left(1-\frac{b(r)}{r}\right)\left(1+r \Phi^{\prime}(r)\right) \dot{f}_{Q}+\frac{f}{2}=-P_{t} .
\end{gathered}
$$

Using (14)-(16), one can explore wormhole geometries in $f(Q)$ gravity. 


\subsection{Energy Conditions}

Energy conditions are some the greatest tools for exploring the geodesic and casual structures of spacetime, as well as for helping in the presentation of physically realistic models. These conditions are developed from the well-known Raychaudhuri equations and can be written as follows [31-33]:

$$
\begin{aligned}
& \frac{d \psi}{d \tau}-\omega_{\mu \nu} \omega^{\mu \nu}+\sigma_{\mu \nu} \sigma^{\mu \nu}+\frac{1}{3} \psi^{2}+R_{\mu v} u^{\mu} u^{v}=0, \\
& \frac{d \psi}{d \tau}-\omega_{\mu \nu} \omega^{\mu \nu}+\sigma_{\mu \nu} \sigma^{\mu v}+\frac{1}{2} \psi^{2}+R_{\mu \nu} \eta^{\mu} \eta^{v}=0 .
\end{aligned}
$$

Here, $\psi, \eta^{\mu}, \omega_{\mu v}$, and $\sigma^{\mu v}$ are the the expansion factor, null vector, rotation, and shear associated with the vector field $u^{\mu}$, respectively. By ignoring the quadratic terms and considering attractive gravity $(\psi<0)$, the above Equations (17) and (18) read as follows:

$$
\begin{aligned}
& R_{\mu \nu} u^{\mu} u^{v} \geq 0 \\
& R_{\mu \nu} \eta^{\mu} \eta^{v} \geq 0 .
\end{aligned}
$$

The energy condition for the distribution of anisotropic fluid matter can be taken from standard General Relativity (GR) as

- $\quad$ Strong energy condition (SEC) if $\rho+P_{j} \geq 0, \rho+\sum_{j} P_{j} \geq 0, \forall j$.

- Weak energy condition (WEC) if $\rho \geq 0, \rho+P_{j} \geq 0, \forall j$.

- Null energy condition (NEC) if $\rho+P_{j} \geq 0, \forall j$.

- Dominant energy condition (DEC) if $\rho \geq 0, \rho \pm P_{j} \geq 0, \forall j$.

Here, $j=r, t$, and $\rho$ and $P_{j}$ describe the energy density and pressure, respectively.

\section{The Study of Traversable Wormholes with Noncommutative Gaussian and Lorentzian Geometries}

In this section, we shall discuss different wormhole geometries with their features. First, we consider the noncommutative Gaussian and Lorentzian geometries with smearing effects in order to calculate the solution for the shape function. The Gaussian and Lorentzian sources of energy density are expressed in (1).

\subsection{Gaussian and Lorentzian Distributions with Linear Symmetric Teleparallel Gravity}

In this subsection, we considered the Lagrangian $f(Q)$ as a linear dependence on $Q$ :

$$
f(Q)=\alpha Q,
$$

where ' $\alpha$ ' is constant. In this model, if we consider $\alpha=1$, then it will be reduced to GR. In addition, for $\alpha=0$, there will be no functional form of this model. Here, in this model, we consider $\alpha<0$ to fulfill the requirements of the energy conditions. The redshift function $\Phi(r)$ in (10) must be finite everywhere. So, in this study, we assume $\Phi(r)=$ constant, i.e., $\Phi^{\prime}(r)=0$. Therefore using Equation (21), the field equations in (14)-(16) are calculated as

$$
\begin{aligned}
\rho & =-\frac{\alpha b^{\prime}(r)}{r^{2}}, \\
p_{r} & =\frac{\alpha b(r)}{r^{3}}, \\
p_{t} & =\alpha\left(\frac{b^{\prime}(r)}{2 r^{2}}-\frac{b(r)}{2 r^{3}}\right) .
\end{aligned}
$$


By comparing Equations (1) and (22), we get the following differential equations based on the Gaussian and Lorentzian distributions:

$$
\begin{aligned}
-\frac{\alpha b^{\prime}(r)}{r^{2}} & =\frac{M e^{-\frac{r^{2}}{4 \Theta}}}{8 \pi^{3 / 2} \Theta^{3 / 2}} \\
-\frac{\alpha b^{\prime}(r)}{r^{2}} & =\frac{\sqrt{\Theta} M}{\pi^{2}\left(\Theta+r^{2}\right)^{2}} .
\end{aligned}
$$

On solving Equation (25), we get an exact shape function for the Gaussian distribution:

$$
b(r)=C_{1}-\frac{M\left(2 \sqrt{\pi} \Theta^{3 / 2} \operatorname{erf}\left(\frac{r}{2 \sqrt{\Theta}}\right)-2 \Theta r e^{-\frac{r^{2}}{4 \Theta}}\right)}{8 \pi^{3 / 2} \alpha \Theta^{3 / 2}}
$$

where $\operatorname{erf}$ and $C_{1}$ represent an error function and a constant of integration, respectively. Here, we consider small values of $r$; if we deal with large values of $r$, then the standard classical behavior will recover. Next, we graphically investigate the profiles of the energy conditions and the shape function. We will choose some particular values of the parameter to satisfy all of the properties of the shape function. Here, we fix $C_{1}=2.5, \Theta=0.5$, and $M=0.0007$.

Figure 1 represents the increasing behavior of the shape function $b(r)$ with respect to $r$. The shape function also follows the flare-out condition, i.e., for $r>r_{0}$, the condition $b^{\prime}(r)<1$ is satisfied. In Figure 2, we show the asymptotic flatness condition, i.e., $\frac{b(r)}{r} \rightarrow 0$ as $r \rightarrow \infty$ is satisfied. In the same figure, we also show $b(r)-r$ with respect to $r$, where one can see the wormhole throat radii for different values of $\alpha$. The WH throats are $r_{0}=2.51$, $r_{0}=2.512, r_{0}=2.517, r_{0}=2.525$, and $r_{0}=2.55$ for $\alpha=-0.005, \alpha=-0.004, \alpha=-0.003$, $\alpha=-0.002$, and $\alpha=-0.001$, respectively. Therefore, all of the results for the shape function are suitable for the construction of a WH in $f(Q)$ gravity.
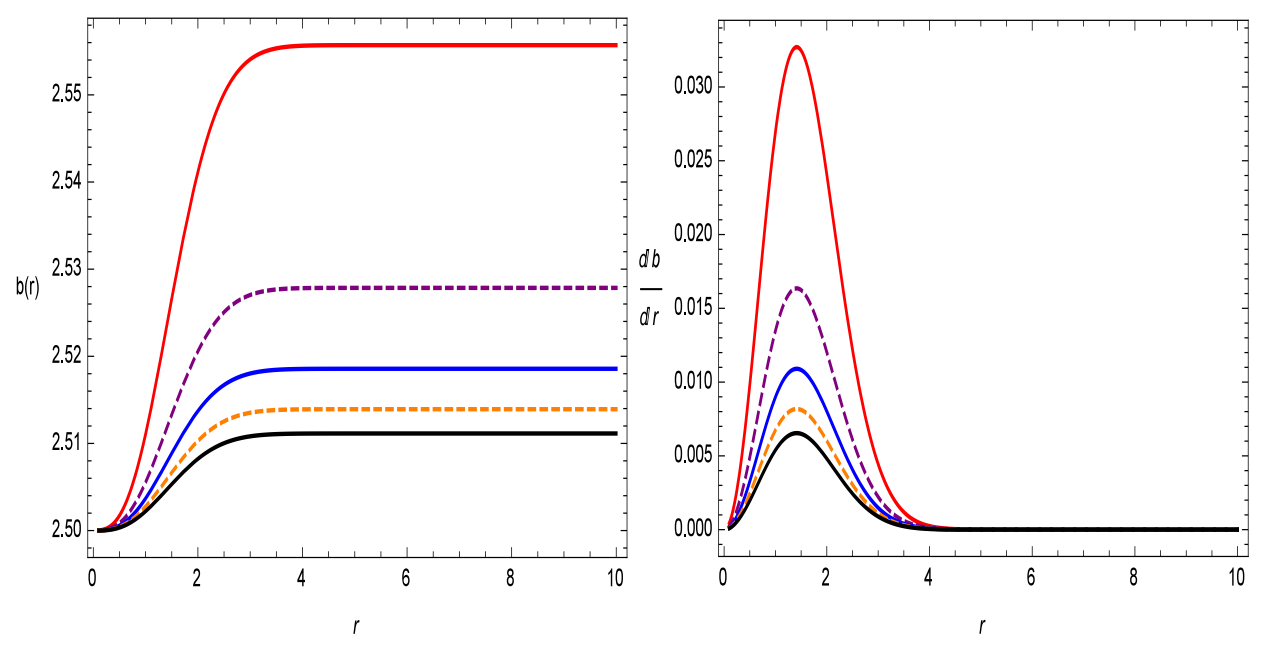

Figure 1. The graphical behavior of $b(r)$ and $b^{\prime}(r)$ for a Gaussian source with $\alpha=-0.001$ $(\star), \alpha=-0.002(\star), \alpha=-0.003(\star), \alpha=-0.004(\star), \alpha=-0.005(\star), C_{1}=2.5, \Theta=0.5$, and $M=0.0007$. 

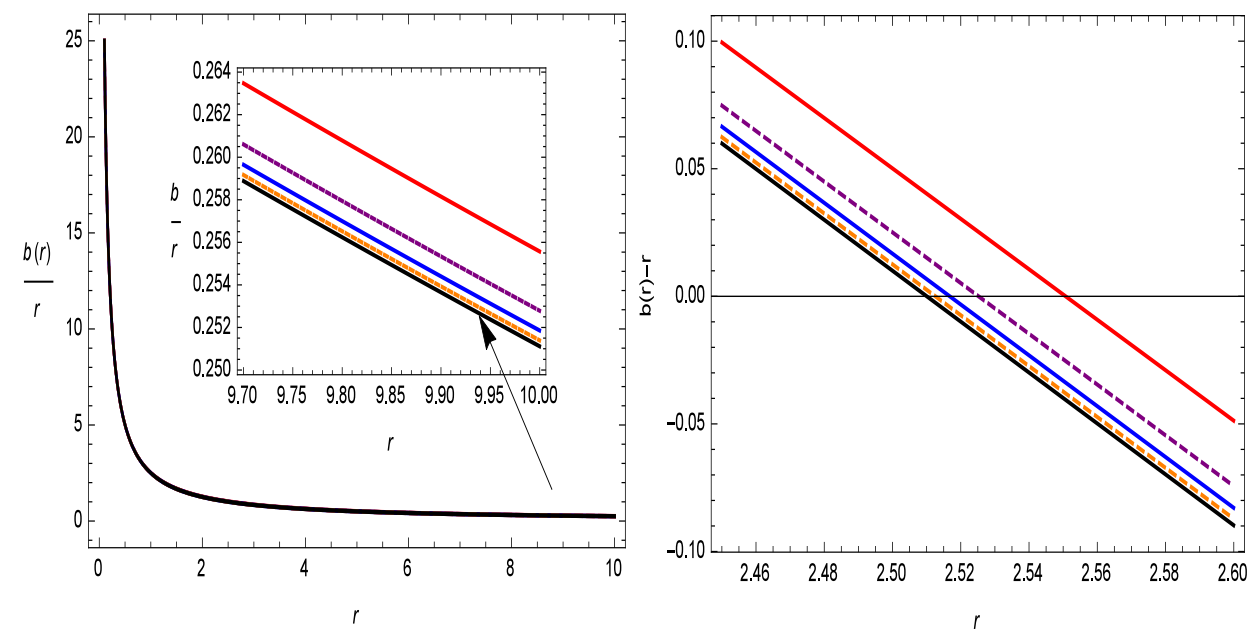

Figure 2. The graphical behavior of $\frac{b(r)}{r}$ and $b(r)-r$ for a Gaussian source with $\alpha=-0.001(\star)$, $\alpha=-0.002(\star), \alpha=-0.003(\star), \alpha=-0.004(\star), \alpha=-0.005(\star), C_{1}=2.5, \Theta=0.5$, and $M=0.0007$.

To investigate the behavior of the energy conditions for the symmetric teleparallel case in the noncommutative background, we use shape function (27) in Equations (22)-(24). Here, we get the following expressions for the energy density, pressure components, and energy conditions under the Gaussian distribution:

$$
\begin{aligned}
& \rho=\frac{M e^{-\frac{r^{2}}{4 \Theta}}}{8 \pi^{3 / 2} \Theta^{3 / 2}}, \\
& p_{r} \quad= \frac{4 \alpha C_{1}-\frac{M e r f\left(\frac{r}{2 \sqrt{\Theta}}\right)}{4}+\frac{M r e^{-\frac{r^{2}}{4 \Theta}}}{\pi^{3 / 2} \sqrt{\Theta}}}{4 r^{3}}, \\
& p_{t} \quad=\frac{2 \sqrt{\pi}\left(\operatorname{Merf}\left(\frac{r}{2 \sqrt{\Theta}}\right)-4 \pi \alpha C_{1}\right)-\frac{M r e^{-\frac{r^{2}}{4 \Theta}\left(2 \Theta+r^{2}\right)}}{\Theta^{3 / 2}}}{16 \pi^{3 / 2} r^{3}}, \\
& \rho+p_{r} \quad=\frac{2 \sqrt{\pi}\left(4 \pi \alpha C_{1}-M e r f\left(\frac{r}{2 \sqrt{\Theta}}\right)\right)+\frac{M r e^{-\frac{r^{2}}{4 \Theta}\left(2 \Theta+r^{2}\right)}}{\Theta^{3 / 2}}}{8 \pi^{3 / 2} r^{3}}, \\
& \rho+p_{t} \quad=\frac{2 \sqrt{\pi}\left(\operatorname{Merf}\left(\frac{r}{2 \sqrt{\Theta}}\right)-4 \pi \alpha C_{1}\right)+\frac{M r e^{-\frac{r^{2}}{4 \Theta}\left(r^{2}-2 \Theta\right)}}{\Theta^{3 / 2}}}{16 \pi^{3 / 2} r^{3}}, \\
& \rho-p_{r} \quad=\frac{2 \sqrt{\pi}\left(\operatorname{Merf}\left(\frac{r}{2 \sqrt{\Theta}}\right)-4 \pi \alpha C_{1}\right)+\frac{M r e^{-\frac{r^{2}}{4 \Theta}\left(r^{2}-2 \Theta\right)}}{\Theta^{3 / 2}}}{8 \pi^{3 / 2} r^{3}}, \\
& \rho-p_{t} \quad=\frac{2 \sqrt{\pi}\left(4 \pi \alpha C_{1}-M e r f\left(\frac{r}{2 \sqrt{\Theta}}\right)\right)+\frac{M r e^{-\frac{r^{2}}{4 \Theta}\left(2 \Theta+3 r^{2}\right)}}{\Theta^{3 / 2}}}{16 \pi^{3 / 2} r^{3}} \\
& \rho+p_{r}+2 p_{t}=0 .
\end{aligned}
$$

The energy conditions are suitable geometric equipment for checking the cosmological models' stability. Hence, this tool was chosen to study the considered models. In addition, the linear Lagrangian form of $f(Q)$ was assumed. Therefore, our cosmological model retains all of the energy conditions of GR. We depict all of the energy conditions for the Gaussian sources in Figures 3-5. In Figure 3, we present the energy density $\rho$ versus $r$, which exhibits a clearly decreasing behavior. One can see in Figure 4 that $\rho+P_{r}<0$ and $\rho+P_{t}>0$, i.e., these are violations of the null energy condition (NEC) for different values 
of $\alpha$. This gives a piece of evidence for the existence of exotic matter at the wormhole's throat, which is essential for a traversable $\mathrm{WH}$. In addition, we depict the profile of the DEC in Figure 5. It can be seen from Equation (35) that strong energy conditions yield $\rho+P_{r}+P_{t}=0$. This is comparable the outcome presented in [34].

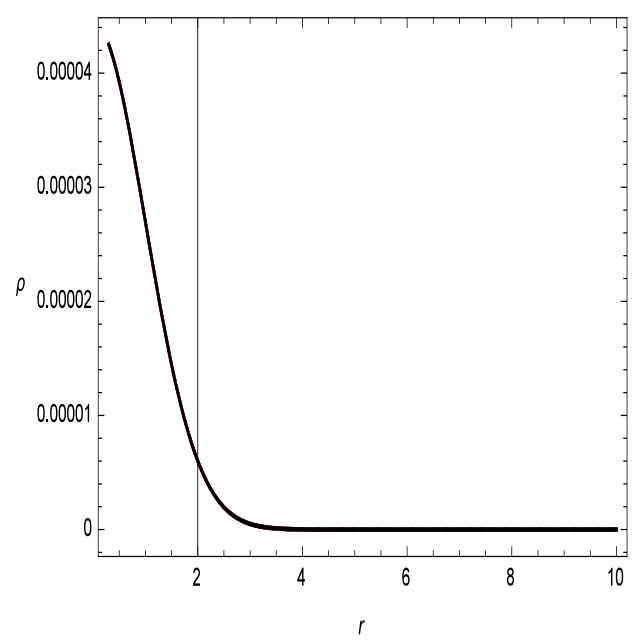

Figure 3. The plot of the energy density $\rho$ with respect to $r$ for a Gaussian source with $\alpha=-0.001(\star), \alpha=-0.002(\star), \alpha=-0.003(\star), \alpha=-0.004(\star), \alpha=-0.005(\star), C_{1}=2.5$, $\Theta=0.5$, and $M=0.0007$.
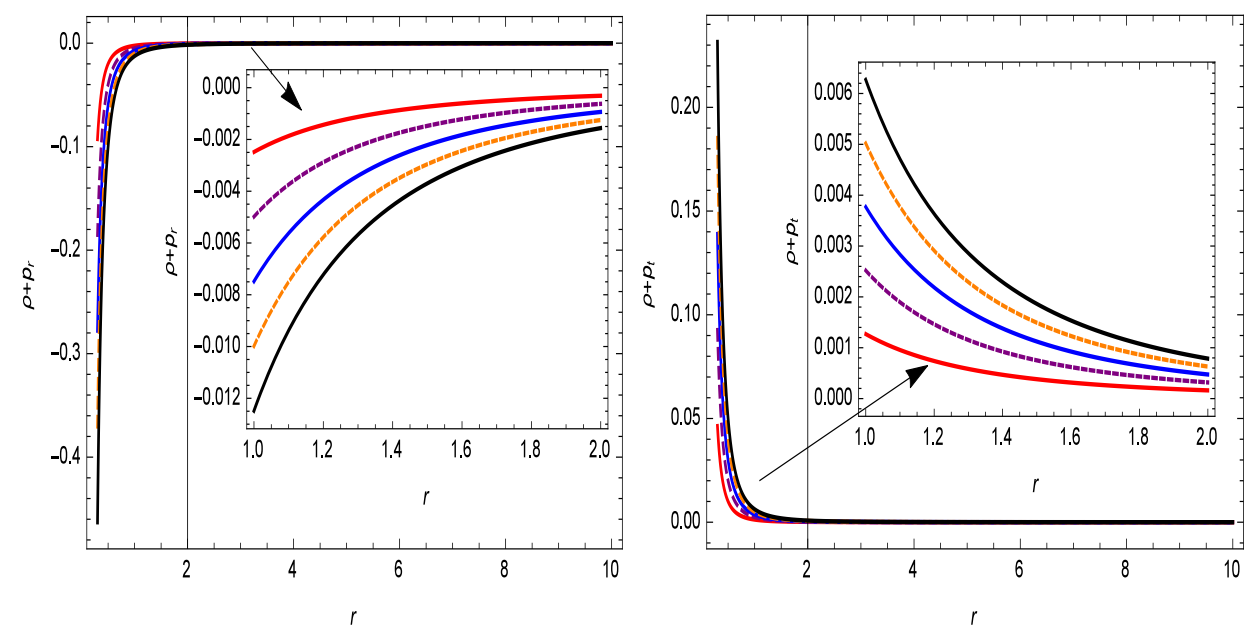

Figure 4. The plots of $\rho+P_{r}$ and $\rho+P_{t}$ with respect to $r$ for a Gaussian source with $\alpha=-0.001(\star)$, $\alpha=-0.002(\star), \alpha=-0.003(\star), \alpha=-0.004(\star), \alpha=-0.005(\star), C_{1}=2.5, \Theta=0.5$, and $M=0.0007$.

On solving Equation (26), we obtain an exact shape function for the Lorentzian distribution:

$$
b(r)=C_{2}-\frac{\sqrt{\Theta} M\left(\frac{\tan ^{-1}\left(\frac{r}{\sqrt{\Theta}}\right)}{2 \sqrt{\Theta}}-\frac{r}{2\left(\Theta+r^{2}\right)}\right)}{\pi^{2} \alpha}
$$

where $C_{2}$ is a constant of integration and $\operatorname{erf}$ represents an error function.

Figure 6 shows the increasing behavior of the shape function $b(r)$ as $r$ increases. To check the legitimacy of the condition $b^{\prime}(r)<1$ for $r>r_{0}$, we plot the first derivative of $b(r)$ in Figure 6. In Figure 7, we plot $\frac{b(r)}{r}$ versus $r$ to verify the asymptotic flatness condition, which gives $\frac{b(r)}{r} \rightarrow 0$ as $r \rightarrow \infty$. In addition, to find the radius of the wormhole's throat, we present $b(r)-r$ versus $r$ in Figure 7 , from which we can see the areas of the 
wormhole's throat for various values of the parameter $\alpha$; these are seen at approximately $r_{0}=2.507, r_{0}=2.509, r_{0}=2.513, r_{0}=2.519$, and $r_{0}=2.537$ for $\alpha=-0.005, \alpha=-0.004$, $\alpha=-0.003, \alpha=-0.002$, and $\alpha=-0.001$, respectively. From Figures 6 and 7 , one can see that the shape function fulfills the necessary conditions for the wormhole in symmetric teleparallel gravity.
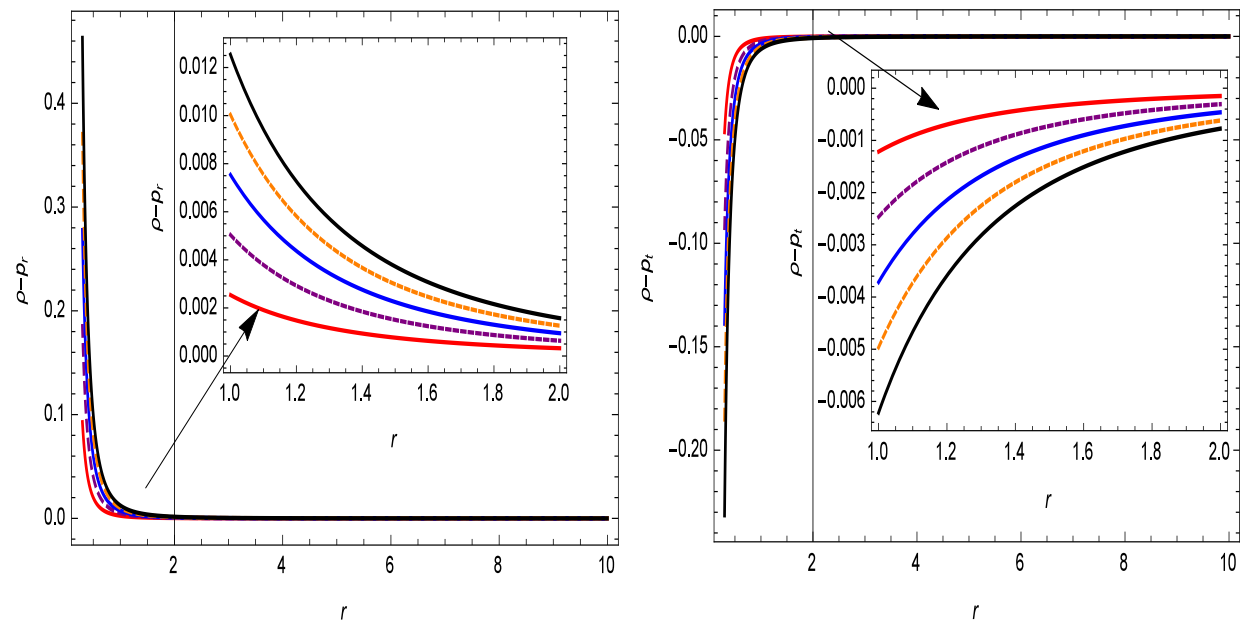

Figure 5. The plots of $\rho-P_{r}$ and $\rho-P_{t}$ with respect to $r$ for a Gaussian source with $\alpha=-0.001(\star)$, $\alpha=-0.002(\star), \alpha=-0.003(\star), \alpha=-0.004(\star), \alpha=-0.005(\star), C_{1}=2.5, \Theta=0.5$, and $M=0.0007$.
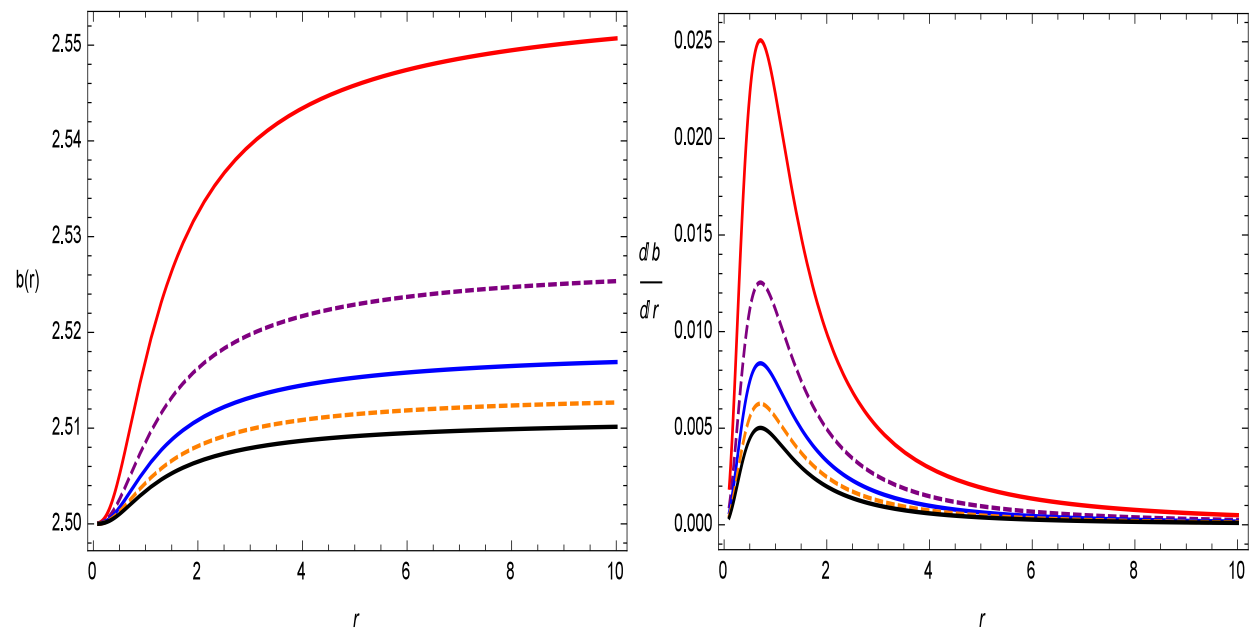

Figure 6. The graphical behavior of $b(r)$ and $b^{\prime}(r)$ for a Lorentzian source with $\alpha=-0.001(\star)$, $\alpha=-0.002(\star), \alpha=-0.003(\star), \alpha=-0.004(\star), \alpha=-0.005(\star), C_{2}=2.5, \Theta=0.5$, and $M=0.0007$. 

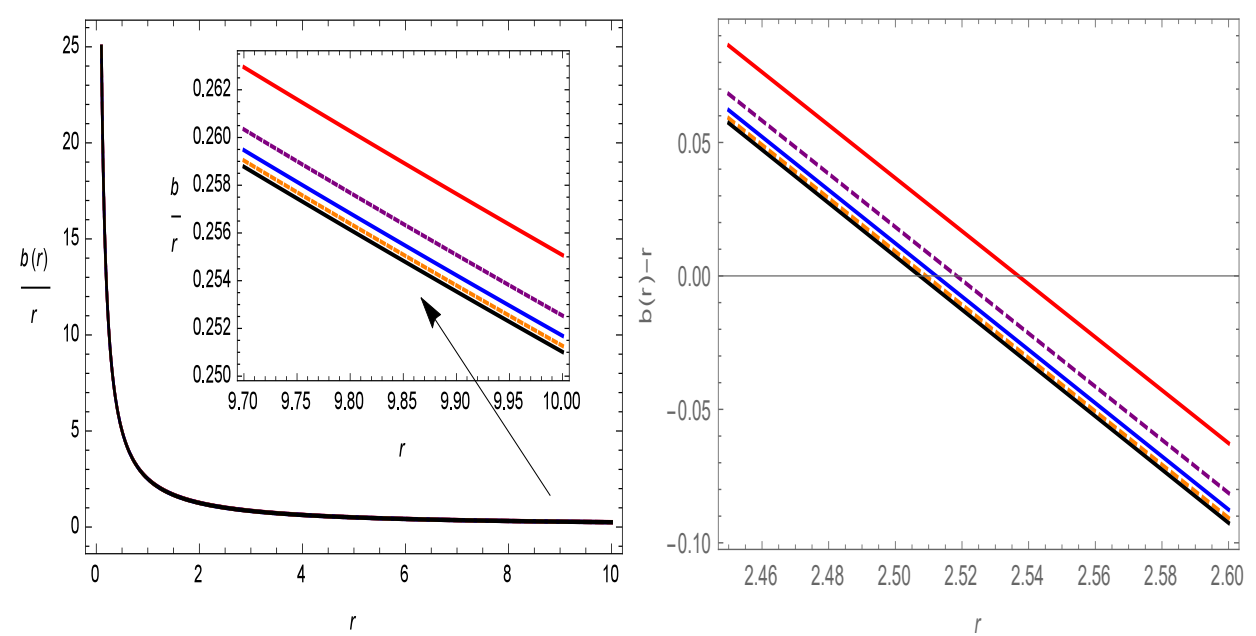

Figure 7. The graphical behavior of $\frac{b(r)}{r}$ and $b(r)-r$ for a Lorentzian source with $\alpha=-0.001(\star)$, $\alpha=-0.002(\star), \alpha=-0.003(\star), \alpha=-0.004(\star), \alpha=-0.005(\star), C_{2}=2.5, \Theta=0.5$, and $M=0.0007$.

By using Equation (36) in Equations (22)-(24), we get the following expressions for the energy density, pressure components, and energy conditions under the Lorentzian distribution:

$$
\begin{aligned}
\rho & =\frac{\sqrt{\Theta} M}{\pi^{2}\left(\Theta+r^{2}\right)^{2}}, \\
p_{r} & =\frac{2 \alpha C_{2}+\frac{\sqrt{\Theta} M r}{\pi^{2}\left(\Theta+r^{2}\right)}-\frac{M \tan ^{-1}\left(\frac{r}{\sqrt{\Theta}}\right)}{2 r^{3}}}{\pi^{2}}, \\
p_{t} & =\frac{-2 \pi^{2} \alpha C_{2}\left(\Theta+r^{2}\right)^{2}-\sqrt{\Theta} M r\left(\Theta+3 r^{2}\right)+M\left(\Theta+r^{2}\right)^{2} \tan ^{-1}\left(\frac{r}{\sqrt{\Theta}}\right)}{4 \pi^{2} r^{3}\left(\Theta+r^{2}\right)^{2}}, \\
\rho+p_{r} & =\frac{2 \pi^{2} \alpha C_{2}\left(\Theta+r^{2}\right)^{2}+\sqrt{\Theta} M r\left(\Theta+3 r^{2}\right)-M\left(\Theta+r^{2}\right)^{2} \tan ^{-1}\left(\frac{r}{\sqrt{\Theta}}\right)}{2 \pi^{2} r^{3}\left(\Theta+r^{2}\right)^{2}}, \\
\rho+p_{t} & =\frac{-2 \pi^{2} \alpha C_{2}\left(\Theta+r^{2}\right)^{2}+\sqrt{\Theta} M r\left(r^{2}-\Theta\right)+M\left(\Theta+r^{2}\right)^{2} \tan ^{-1}\left(\frac{r}{\sqrt{\Theta}}\right)}{4 \pi^{2} r^{3}\left(\Theta+r^{2}\right)^{2}}, \\
\rho-p_{r} & =\frac{-2 \pi^{2} \alpha C_{2}\left(\Theta+r^{2}\right)^{2}+\sqrt{\Theta} M r\left(r^{2}-\Theta\right)+M\left(\Theta+r^{2}\right)^{2} \tan ^{-1}\left(\frac{r}{\sqrt{\Theta}}\right)}{2 \pi^{2} r^{3}\left(\Theta+r^{2}\right)^{2}}, \\
\rho-p_{t} & =\frac{2 \pi^{2} \alpha C_{2}\left(\Theta+r^{2}\right)^{2}+\sqrt{\Theta} M r\left(\Theta+7 r^{2}\right)-M\left(\Theta+r^{2}\right)^{2} \tan ^{-1}\left(\frac{r}{\sqrt{\Theta}}\right)}{4 \pi^{2} r^{3}\left(\Theta+r^{2}\right)^{2}}, \\
\rho+p_{r}+2 p_{t} \quad= & 0 .
\end{aligned}
$$

We show the graphical behavior of the energy conditions under the Lorentzian source in Figures 8-10. In Figure 8, we present the energy density $\rho$ versus $r$, which exhibits a clearly decreasing behavior throughout the spacetime. From Figure 9, we can observe that the NEC is violated because $\rho+P_{r}<0$. Subsequently, it can be determined that the wormhole solutions that have been procured are acceptable in this $f(Q)$ gravity. In addition, we present a graph of the DEC in Figure 10. From Equation (44), we can see that the strong energy condition (SEC) yields $\rho+P_{r}+P_{t}=0$. This is comparable to the outcome presented in [34]. 


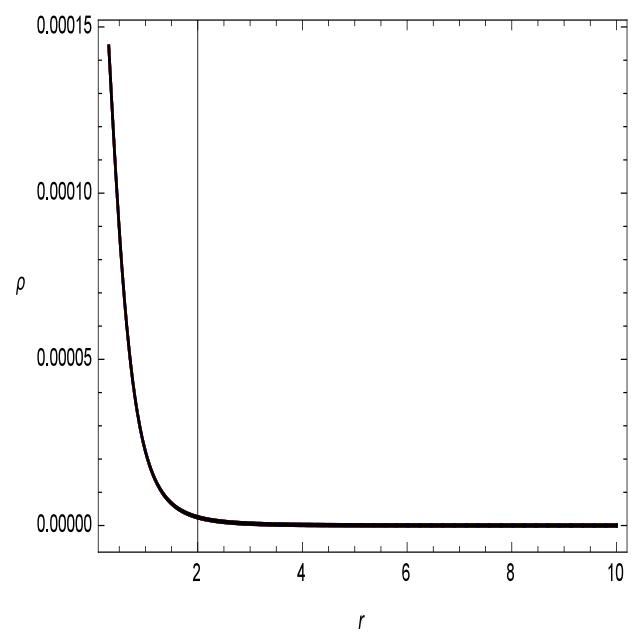

Figure 8. The plot of the energy density $\rho$ with respect to $r$ for a Lorentzian source with $\alpha=-0.001(\star), \alpha=-0.002(\star), \alpha=-0.003(\star), \alpha=-0.004(\star), \alpha=-0.005(\star), C_{2}=2.5$, $\Theta=0.5$, and $M=0.0007$.
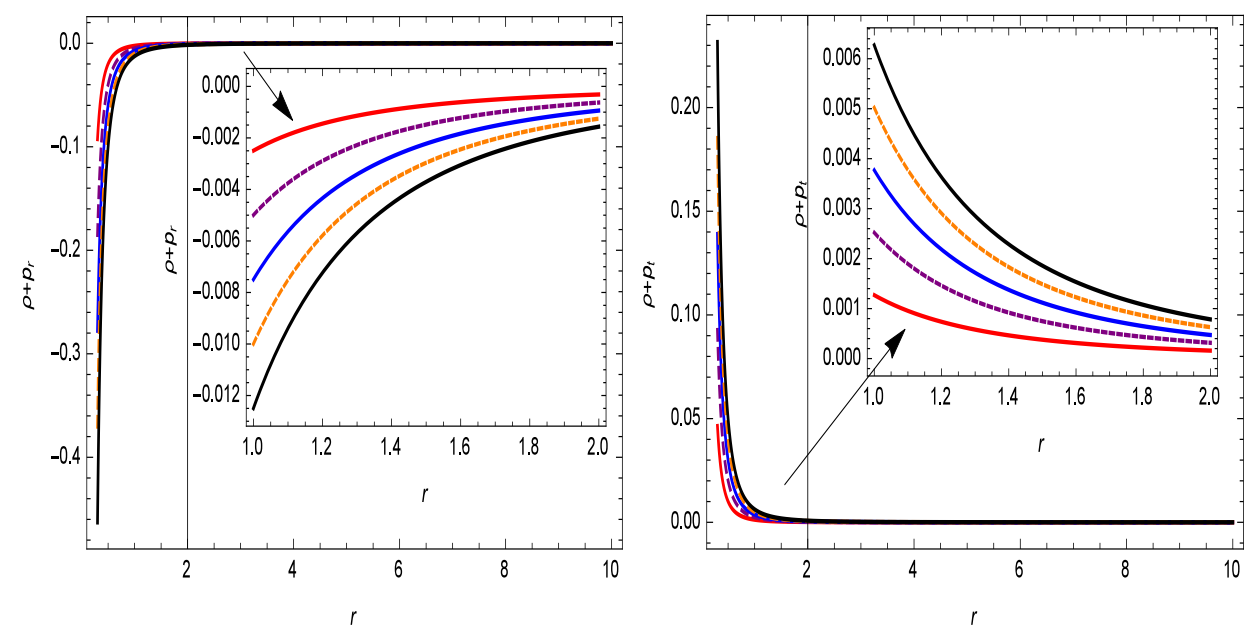

Figure 9. The plots of $\rho+P_{r}$ and $\rho+P_{t}$ with respect to $r$ for a Lorentzian source with $\alpha=-0.001(\star)$, $\alpha=-0.002(\star), \alpha=-0.003(\star), \alpha=-0.004(\star), \alpha=-0.005(\star), C_{2}=2.5, \Theta=0.5$, and $M=0.0007$. 

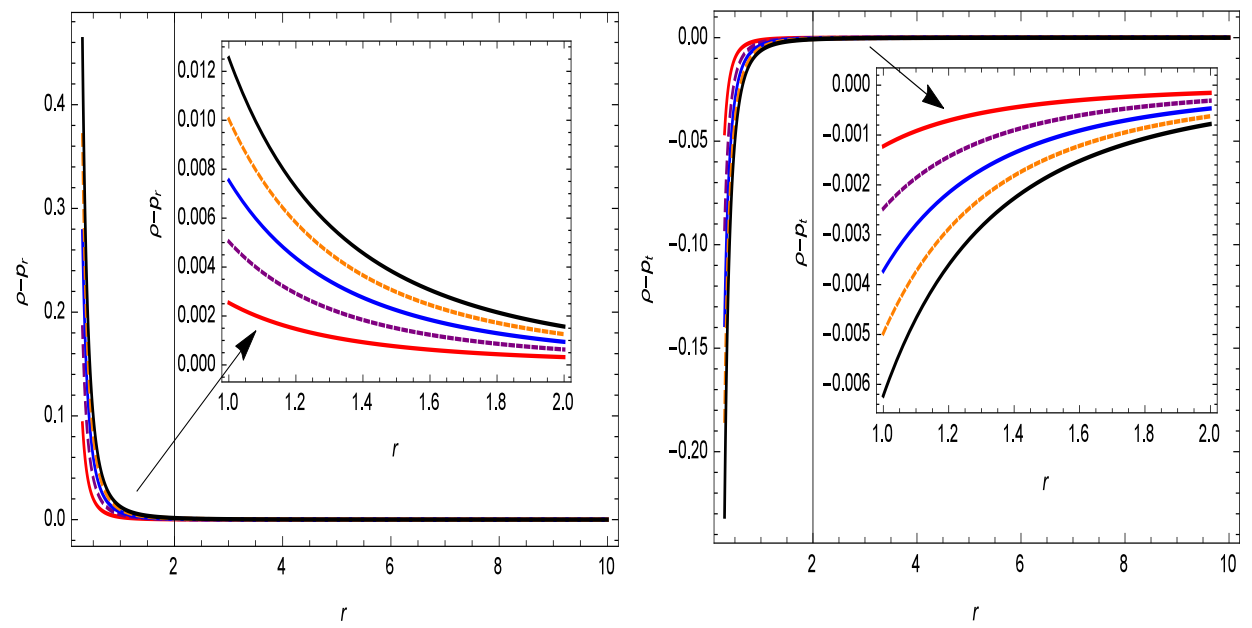

Figure 10. The plots of $\rho-P_{r}$ and $\rho-P_{t}$ with respect to $r$ for a Lorentzian source with $\alpha=-0.001(\star), \alpha=-0.002(\star), \alpha=-0.003(\star), \alpha=-0.004(\star), \alpha=-0.005(\star), C_{2}=2.5$, $\Theta=0.5$, and $M=0.0007$.

\subsection{Gaussian and Lorentzian Distributions with Exponential Symmetric Teleparallel Gravity}

Here, we assume a specific form of the exponential $f(Q)$ model [35]:

$$
f(Q)=Q-\alpha\left(1-e^{-Q}\right)
$$

where ' $\alpha$ ' is constant. In this model, if we consider $\alpha=0$, then it will be reduced to GR. Here, we choose $\alpha>0$ to fulfill all requirements of the energy conditions. In this study, we consider $\Phi(r)=$ constant, i.e., $\Phi^{\prime}(r)=0$. Therefore, the field equations in (14)-(16) read:

$$
\begin{aligned}
& \rho=\frac{4}{r^{5}} \alpha\left(1-\frac{b(r)}{r}\right) e^{\frac{2}{r^{2}}\left(1-\frac{b(r)}{r}\right)}\left(-3 b(r)+r b^{\prime}(r)+2 r\right)- \\
& \frac{1}{r}\left(-\frac{1}{r}+\frac{b(r)+r b^{\prime}(r)}{r^{2}}\right)\left(1-\alpha e^{\frac{2}{r^{2}}\left(1-\frac{b(r)}{r}\right)}\right)-\frac{1}{2}\left(\frac{2}{r^{2}}\left(1-\frac{b(r)}{r}\right)+\alpha\left(1-e^{\frac{2}{r^{2}}\left(1-\frac{b(r)}{r}\right)}\right)\right) \\
& P_{r}=\frac{1}{2}\left(\frac{2}{r^{2}}\left(1-\frac{b(r)}{r}\right)+\alpha\left(1-e^{\frac{2}{r^{2}}\left(1-\frac{b(r)}{r}\right)}\right)\right)-\left(1-\alpha e^{\frac{2}{r^{2}}\left(1-\frac{b(r)}{r}\right)}\right)\left(-\frac{1}{r^{2}}+\frac{2}{r^{2}}\left(1-\frac{b(r)}{r}\right)\right) \\
& P_{t}=\frac{1}{2}\left(\frac{2}{r^{2}}\left(1-\frac{b(r)}{r}\right)+\alpha\left(1-e^{\frac{2}{r^{2}}\left(1-\frac{b(r)}{r}\right)}\right)\right)- \\
& \frac{2}{r^{5}} \alpha\left(1-\frac{b(r)}{r}\right) e^{\frac{2}{r^{2}}\left(1-\frac{b(r)}{r}\right)}\left(-3 b(r)+r b^{\prime}(r)+2 r\right)- \\
& \frac{1}{r}\left(1-\alpha e^{\frac{2}{r^{2}}\left(1-\frac{b(r)}{r}\right)}\right)\left(\frac{1}{r}\left(1-\frac{b(r)}{r}\right)-\frac{r b^{\prime}(r)-b(r)}{2 r^{2}}\right)
\end{aligned}
$$

By comparing Equations (1) and (46), we get the following differential equations based on the Gaussian and Lorentzian distributions:

$$
\begin{aligned}
& \frac{4}{r^{5}} \alpha\left(1-\frac{b(r)}{r}\right) e^{\frac{2}{r^{2}}\left(1-\frac{b(r)}{r}\right)}\left(-3 b(r)+r b^{\prime}(r)+2 r\right) \\
& -\frac{1}{r}\left(-\frac{1}{r}+\frac{b(r)+r b^{\prime}(r)}{r^{2}}\right)\left(1-\alpha e^{\frac{2}{r^{2}}\left(1-\frac{b(r)}{r}\right)}\right) \\
& -\frac{1}{2}\left(\frac{2}{r^{2}}\left(1-\frac{b(r)}{r}\right)+\alpha\left(1-e^{\frac{2}{r^{2}}\left(1-\frac{b(r)}{r}\right)}\right)\right)=\frac{M e^{-\frac{r^{2}}{4 \Theta}}}{8 \pi^{3 / 2} \Theta^{3 / 2}},
\end{aligned}
$$




$$
\begin{aligned}
& \frac{4}{r^{5}} \alpha\left(1-\frac{b(r)}{r}\right) e^{\frac{2}{r^{2}}\left(1-\frac{b(r)}{r}\right)}\left(-3 b(r)+r b^{\prime}(r)+2 r\right) \\
& -\frac{1}{r}\left(-\frac{1}{r}+\frac{b(r)+r b^{\prime}(r)}{r^{2}}\right)\left(1-\alpha e^{\frac{2}{r^{2}}\left(1-\frac{b(r)}{r}\right)}\right) \\
& -\frac{1}{2}\left(\frac{2}{r^{2}}\left(1-\frac{b(r)}{r}\right)+\alpha\left(1-e^{\left.\left.\frac{2}{r^{2}}\left(1-\frac{b(r)}{r}\right)\right)\right)=\frac{\sqrt{\Theta} M}{\pi^{2}\left(\Theta+r^{2}\right)^{2}} .}\right.\right.
\end{aligned}
$$

Due to the high linearity in Equations (49) and (50), we numerically inspect the shape function and energy conditions for the wormhole solution. Here, we use the initial condition of $b(0.2)=4.7$.

Here, we assume the parameter $\alpha \in(0, \infty)$, and we find some particular qualities inside this range. It can be seen from the graphs that for the various estimations of $\alpha$, the results change. The graphical behavior of the shape functions for the Gaussian and Lorentzian sources is shown in Figures 11-14. In the left panels of Figures 11 and 13, we plot the shape function $b(r)$ versus $r$ to show the increasing behavior of the shape function. In the right panels of Figures 11 and 13, we plot the first derivative of $b(r)$ with respect to $r$ in order to inspect the flare-out condition, which indicates that $b^{\prime}\left(r_{0}\right)<1$ at $r=r_{0}$ for distinct estimates of $\alpha$. To examine the flatness condition, we plot $\frac{b(r)}{r}$ with respect to $r$ in Figures 12 and 14, which indicate that it approaches zero as $r$ tends to infinity. We plot $b(r)-r$ versus $r$ in the same figures to locate the wormhole's throat. Interestingly, it was found that the values of the wormhole's throat are the same under the Gaussian- and Lorentzian-distributed frameworks. For $\alpha=0.005, b(r)-r$ crosses the $r$-axis at $r_{0}=4.722$, which is the wormhole's throat. Similarly, the throat radii are located at $r_{0}=4.736,4.753,4.771$, and 4.788 for $\alpha=0.010,0.015,0.020$, and 0.025 , respectively, under the Gaussian and Lorentzian distributions.

The behavior of the energy conditions for specific exponential symmetric teleparallel gravity under the Gaussian and Lorentzian distributions is shown in Figures 15-20. Figures 17 and 20 show the plots of the energy density $\rho$ versus $r$, indicating the clearly decreasing behavior. In Figures 16 and 19, we show the behavior of the DEC. It is visible from Figures 15 and 18 that the NEC is violated due to the negative behavior of $\rho+P_{r}$. Consequently, it can be determined that the wormhole solutions that have been procured are acceptable in this symmetric teleparallel gravity.
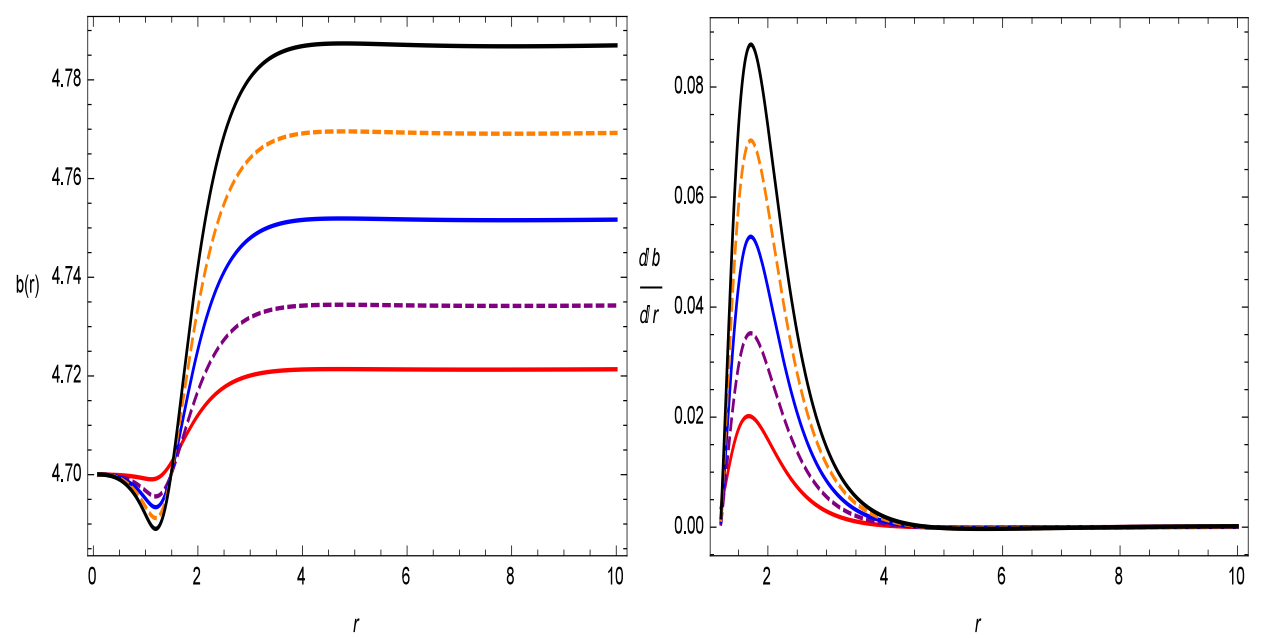

Figure 11. The graphical behavior of $b(r)$ and $b^{\prime}(r)$ for a Gaussian source with $\alpha=0.005(\star)$, $\alpha=0.010(\star), \alpha=0.015(\star), \alpha=0.020(\star), \alpha=0.025(\star), \Theta=0.5$, and $M=0.0007$. 

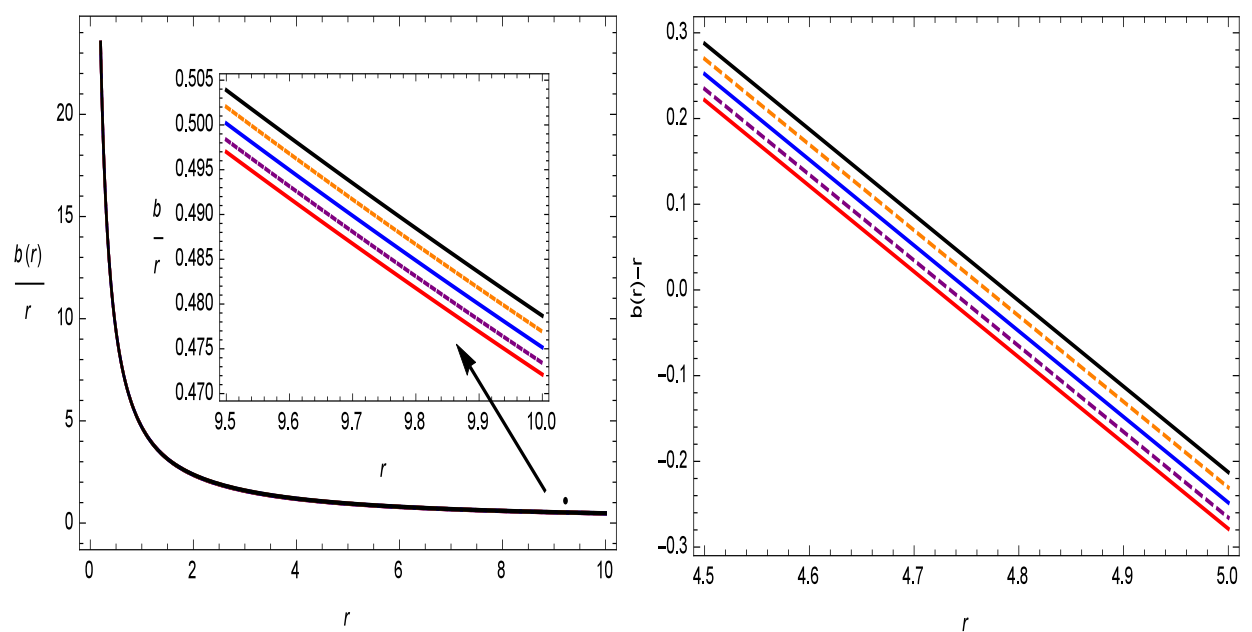

Figure 12. The graphical behavior of $\frac{b(r)}{r}$ and $b(r)-r$ for a Gaussian source with $\alpha=0.005(\star)$, $\alpha=0.010(\star), \alpha=0.015(\star), \alpha=0.020(\star), \alpha=0.025(\star), \Theta=0.5$, and $M=0.0007$.
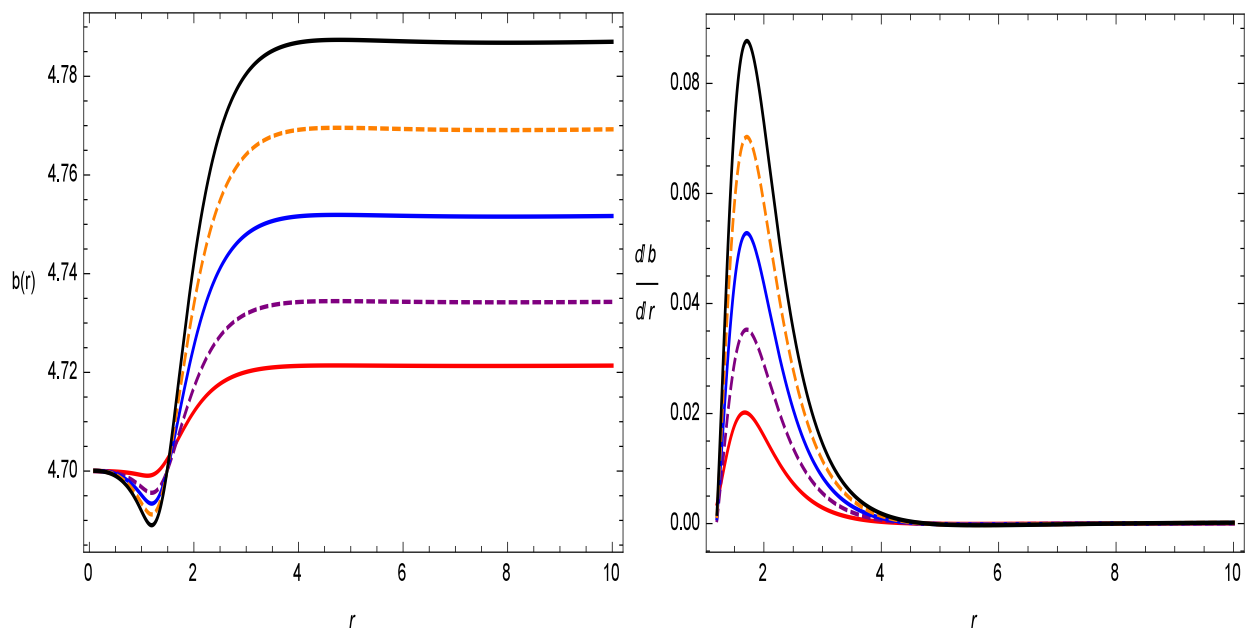

Figure 13. The graphical behavior of $b(r)$ and $b^{\prime}(r)$ for a Lorentzian source with $\alpha=0.005(\star)$, $\alpha=0.010(\star), \alpha=0.015(\star), \alpha=0.020(\star), \alpha=0.025(\star), \Theta=0.5$, and $M=0.0007$.
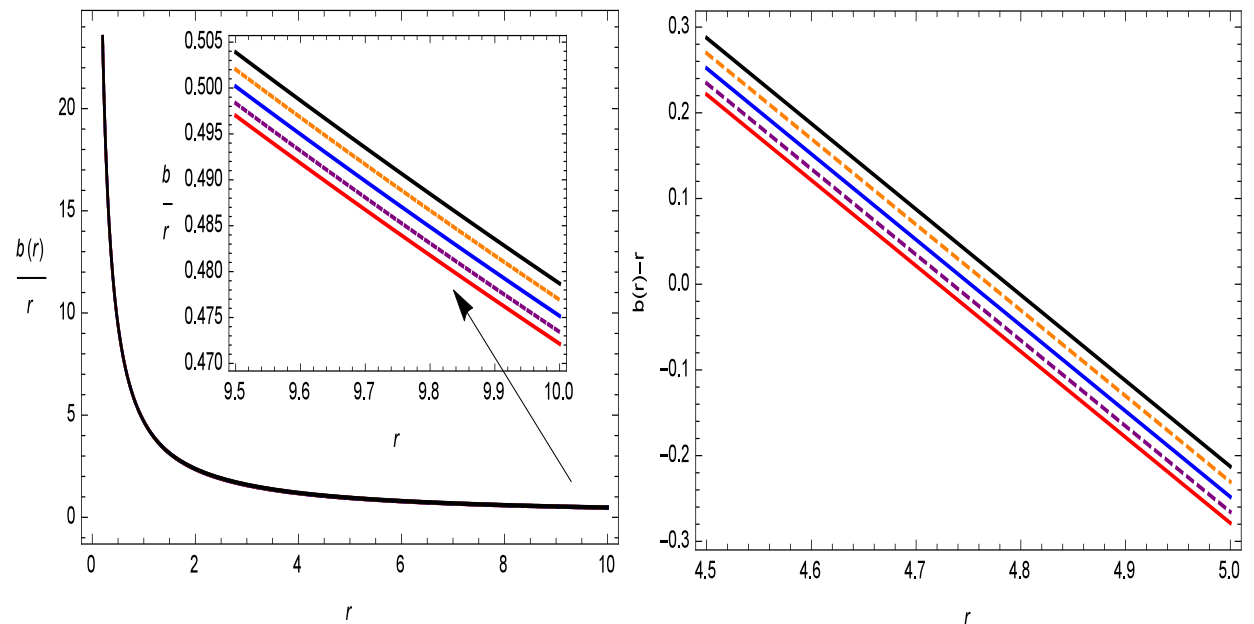

Figure 14. The graphical behavior of $\frac{b(r)}{r}$ and $b(r)-r$ for a Lorentzian source with $\alpha=0.005(\star)$, $\alpha=0.010(\star), \alpha=0.015(\star), \alpha=0.020(\star), \alpha=0.025(\star), \Theta=0.5$, and $M=0.0007$. 

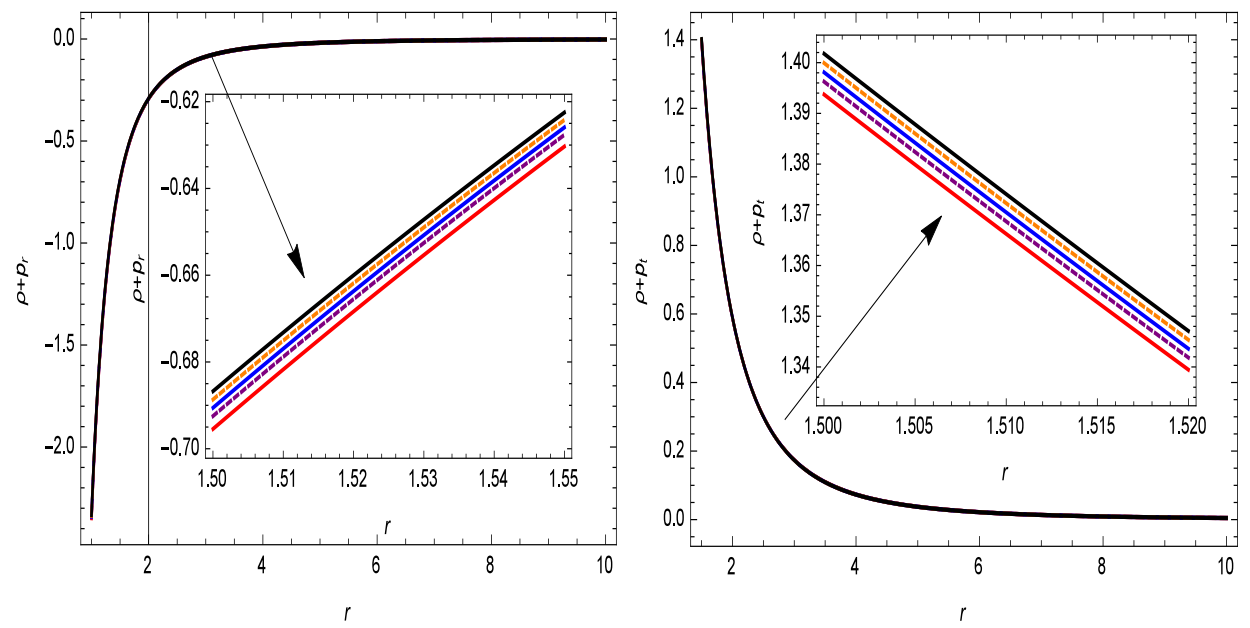

Figure 15. The plots of $\rho+P_{r}$ and $\rho+P_{t}$ with respect to $r$ for a Gaussian source with $\alpha=0.005(\star)$, $\alpha=0.010(\star), \alpha=0.015(\star), \alpha=0.020(\star), \alpha=0.025(\star), \Theta=0.5$, and $M=0.0007$.
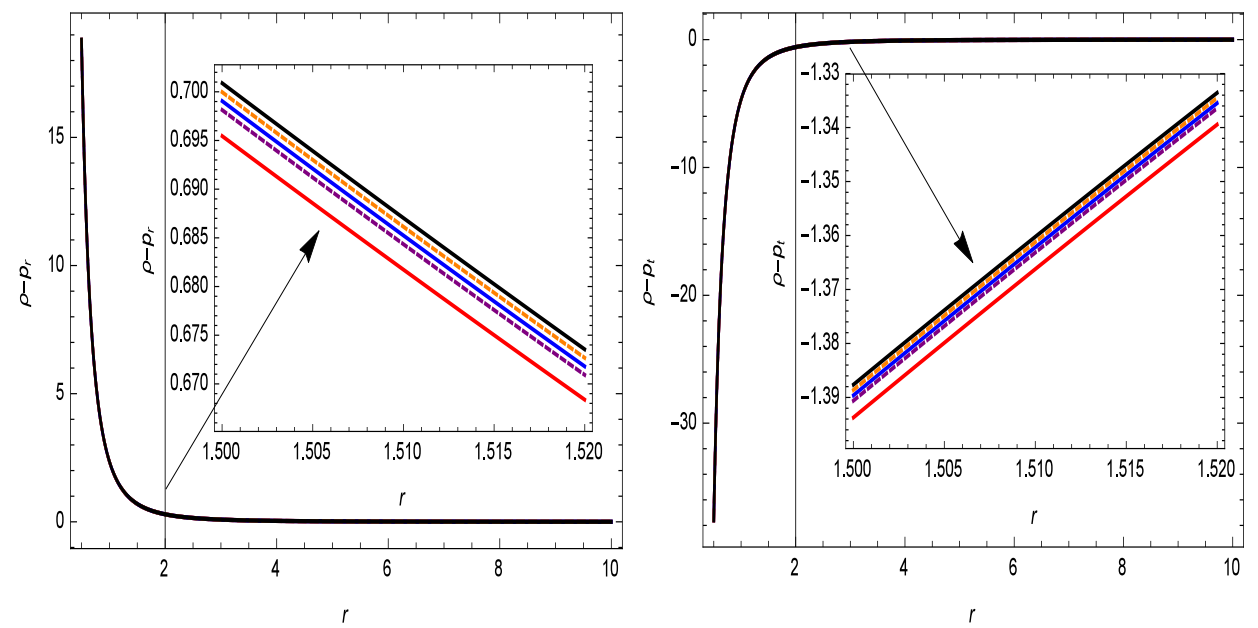

Figure 16. The plots of $\rho-P_{r}$ and $\rho-P_{t}$ with respect to $r$ for a Gaussian source with $\alpha=0.005$ ( $\star$ ), $\alpha=0.010(\star), \alpha=0.015(\star), \alpha=0.020(\star), \alpha=0.025(\star), \Theta=0.5$, and $M=0.0007$.

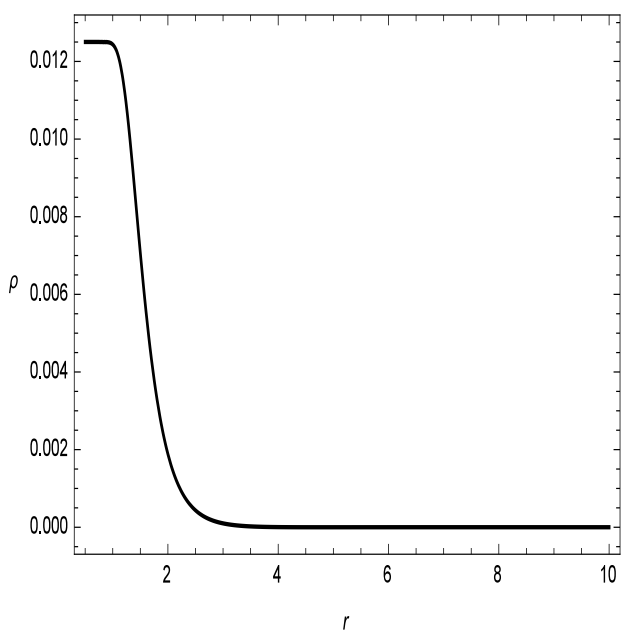

Figure 17. The plot of the energy density $\rho$ with respect to $r$ for a Gaussian source with $\alpha=0.005(\star)$, $\alpha=0.010(\star), \alpha=0.015(\star), \alpha=0.020(\star), \alpha=0.025(\star), \Theta=0.5$, and $M=0.0007$. 

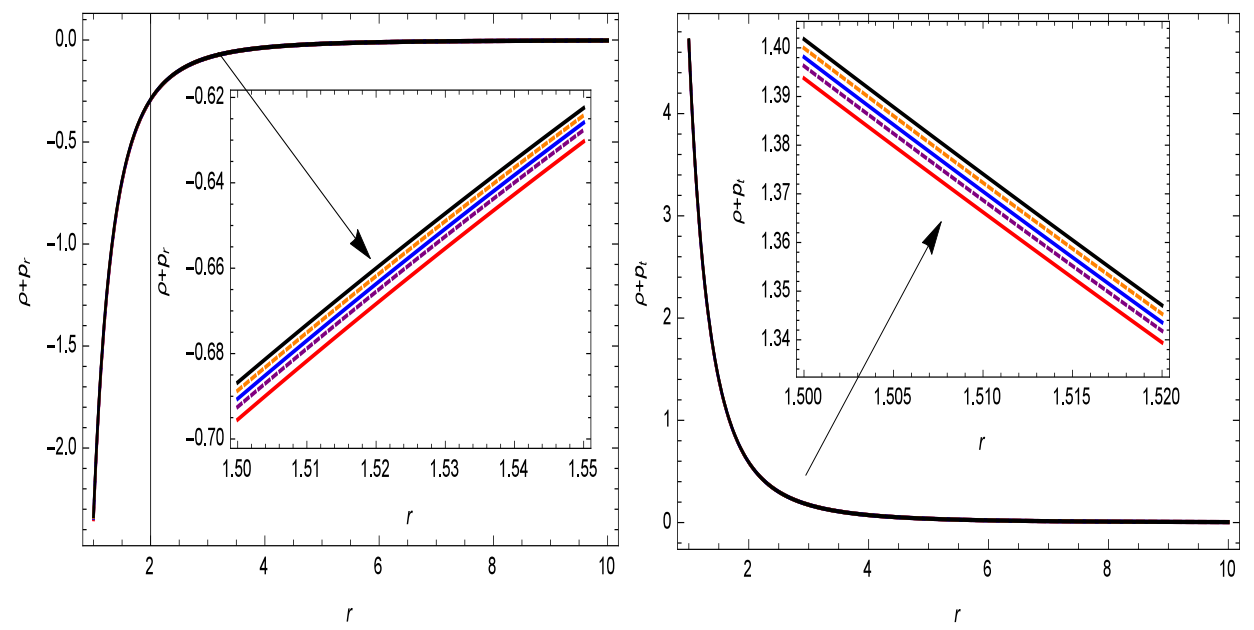

Figure 18. The plots of $\rho+P_{r}$ and $\rho+P_{t}$ with respect to $r$ for a Lorentzian source with $\alpha=0.005(\star)$, $\alpha=0.010(\star), \alpha=0.015(\star), \alpha=0.020(\star), \alpha=0.025(\star), \Theta=0.5$, and $M=0.0007$.
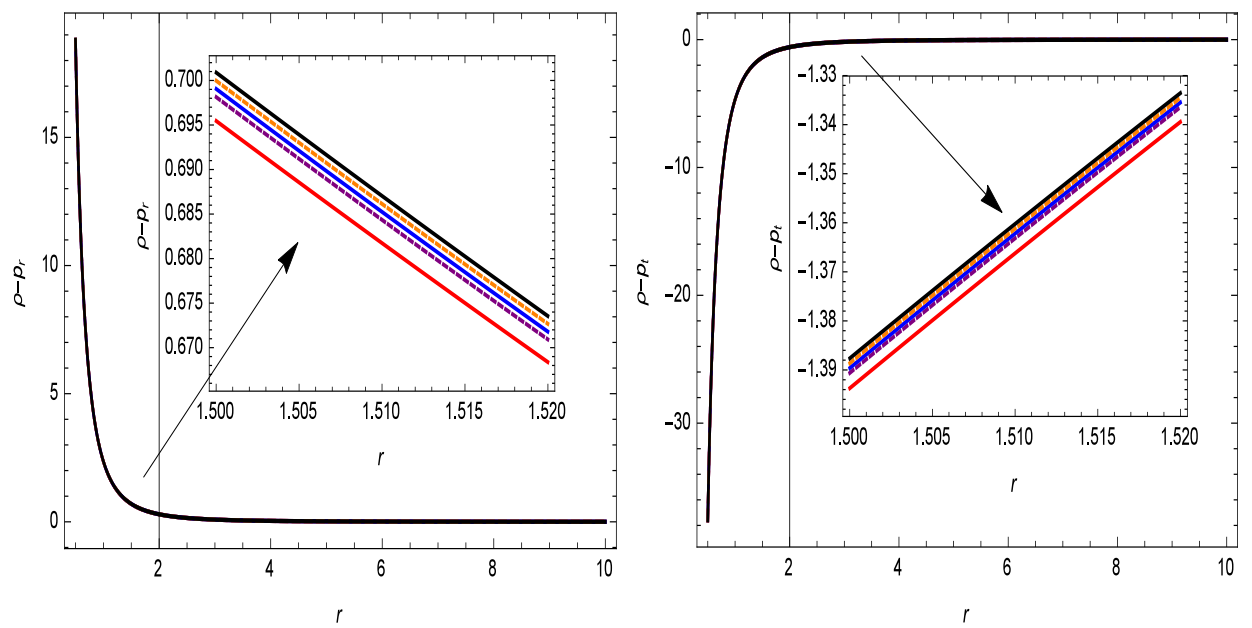

Figure 19. The plots of $\rho-P_{r}$ and $\rho-P_{t}$ with respect to $r$ for a Lorentzian source with $\alpha=0.005(\star)$, $\alpha=0.010(\star), \alpha=0.015(\star), \alpha=0.020(\star), \alpha=0.025(\star), \Theta=0.5$, and $M=0.0007$.

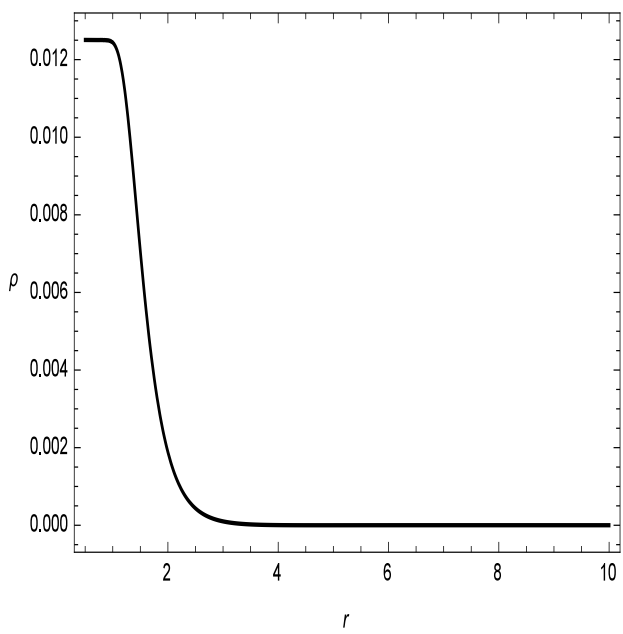

Figure 20. The plot of the energy density $\rho$ with respect to $r$ for a Lorentzian source with $\alpha=0.005(\star), \alpha=0.010(\star), \alpha=0.015(\star), \alpha=0.020(\star), \alpha=0.025(\star), \Theta=0.5$, and $M=0.0007$. 


\section{Stability of the Gaussian and Lorentzian Wormhole Models}

Here, we use the generalized Tolman-Oppenheimer-Volkov (TOV) equation [36-38] to find the stability of the wormhole solution in the Gaussian and Lorentzian distributions in noncommutative backgrounds. The generalized TOV equation can be written as

$$
\frac{\omega^{\prime}}{2}\left(\rho+p_{r}\right)+\frac{d p_{r}}{d r}+\frac{2}{r}\left(p_{r}-p_{t}\right)=0,
$$

where $\omega=2 \Phi(r)$.

Due to the distribution of anisotropic matter, the hydrostatic, gravitational, and anisotropic forces are defined as follows:

$$
F_{h}=-\frac{d p_{r}}{d r}, \quad F_{g}=-\frac{\mathcal{W}^{\prime}}{2}\left(\rho+p_{r}\right), \quad F_{a}=\frac{2}{r}\left(p_{t}-p_{r}\right) .
$$

To find the equilibrium of the wormhole solutions, it is necessary for $F_{h}+F_{g}+F_{a}=0$ to hold. Because we assumed that the redshift function $\Phi(r)=$ constant in this study, the gravitational contribution $F_{g}$ will vanish in the equilibrium equation. Hence, the equilibrium equation becomes

$$
F_{h}+F_{a}=0 .
$$

Using Equations (29) and (30), we get the following equations for the hydrostatic and anisotropic forces under the Gaussian distribution for the linear model:

$$
\begin{aligned}
& F_{a}=\frac{6 \sqrt{\pi}\left(\operatorname{Merf}\left(\frac{r}{2 \sqrt{\Theta}}\right)-4 \pi \alpha C_{1}\right)-\frac{M r e^{-\frac{r^{2}}{4 \Theta}\left(6 \Theta+r^{2}\right)}}{\Theta^{3 / 2}}}{8 \pi^{3 / 2} r^{4}}, \\
& F_{h}=\frac{6 \sqrt{\pi}\left(4 \pi \alpha C_{1}-\operatorname{Merf}\left(\frac{r}{2 \sqrt{\Theta}}\right)\right)+\frac{M r e^{-\frac{r^{2}}{4 \Theta}\left(6 \Theta+r^{2}\right)}}{\Theta^{3 / 2}}}{8 \pi^{3 / 2} r^{4}} .
\end{aligned}
$$

Again, using Equations (38) and (39), we get the following equations for the hydrostatic and anisotropic forces under the Lorentzian distribution for the linear model:

$$
\begin{aligned}
& F_{a}=-\frac{6 \pi^{2} \alpha C_{2}\left(\Theta+r^{2}\right)^{2}+\sqrt{\Theta} M r\left(3 \Theta+5 r^{2}\right)-3 M\left(\Theta+r^{2}\right)^{2} \tan ^{-1}\left(\frac{r}{\sqrt{\Theta}}\right)}{2 \pi^{2} r^{4}\left(\Theta+r^{2}\right)^{2}}, \\
& F_{h}=\frac{6 \sqrt{\pi}\left(4 \pi \alpha C_{1}-M e r f\left(\frac{r}{2 \sqrt{\Theta}}\right)\right)+\frac{M r e^{-\frac{r^{2}}{4 \Theta}\left(6 \Theta+r^{2}\right)}}{\Theta^{3 / 2}} .}{8 \pi^{3 / 2} r^{4}} .
\end{aligned}
$$

Figures 21 and 22 represent the graphs of the hydrostatic and anisotropic forces of the wormhole solutions for the linear and exponential models under the Gaussian and Lorentzian distributions. In the left panel of Figure 21, one can see the graph of the anisotropic and hydrostatic forces for the Gaussian distribution; in the graph in the right panel of Figure 21, one can connect this to the Lorentzian distribution for the linear model. It is visible in Figure 21 that the behaviors of the anisotropic and hydrostatic forces are similar yet inverse to one another; hence, they cancel each other out to satisfy Equation (53). Similarly, the left panel and right panel of Figure 22 show the graphs of the anisotropic and hydrostatic forces for the Gaussian and Lorentzian sources, respectively, for the exponential model. Figure 22 depicts that the anisotropic and hydrostatic forces' behaviors are similar but opposite to one another; hence, they cancel each other's effects. This balanced development of the anisotropic and hydrostatic forces shows that the wormholes that we obtained are stable. 

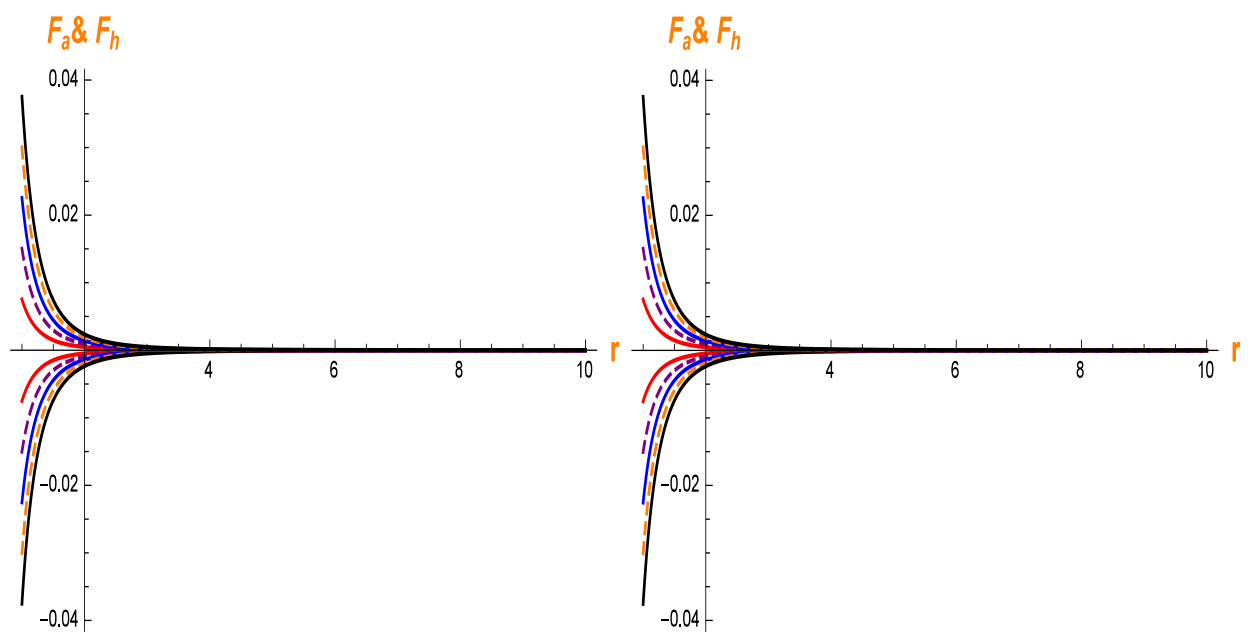

Figure 21. Plots of $F_{a}$ and $F_{h}$ with (left) the Gaussian distribution and (right) the Lorentzian distribution with the linear model for $\alpha=-0.001(\star), \alpha=-0.002(\star), \alpha=-0.003(\star), \alpha=-0.004(\star)$, $\alpha=-0.005(\star), C_{1}=C_{2}=2.5, \Theta=0.5$, and $M=0.0007$.
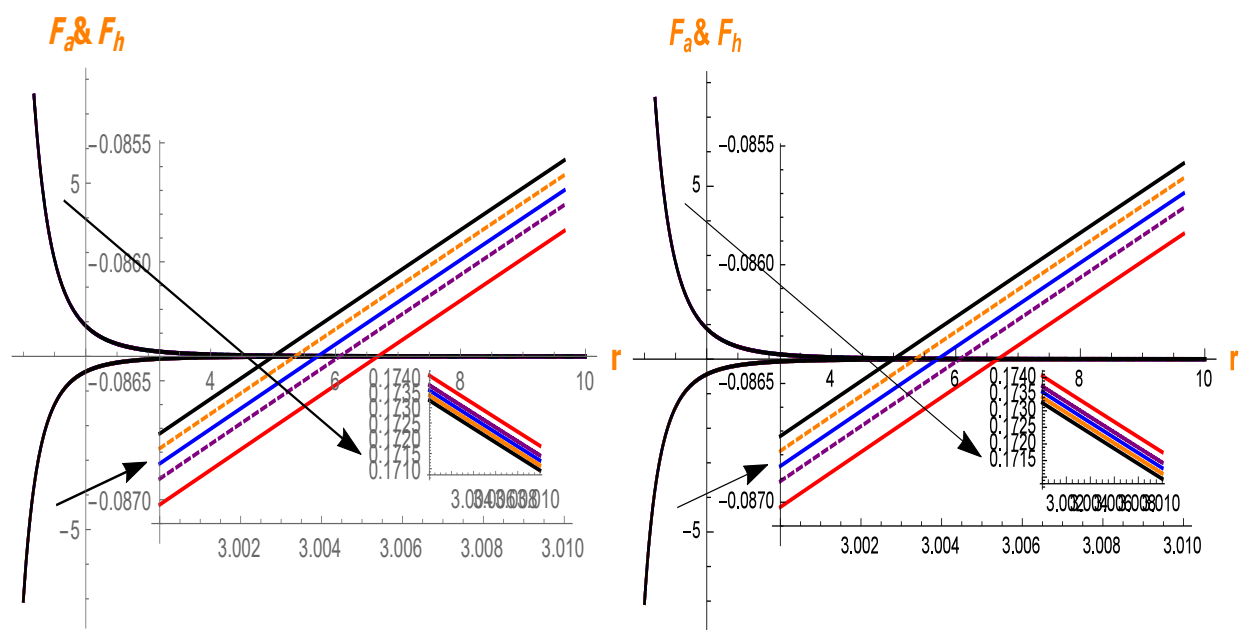

Figure 22. Plots of $F_{a}$ and $F_{h}$ with (left) the Gaussian distribution and (right) the Lorentzian distribution with the exponential model for $\alpha=0.005(\star), \alpha=0.010(\star), \alpha=0.015(\star), \alpha=0.020(\star)$, $\alpha=0.025(\star), \Theta=0.5$, and $M=0.0007$.

\section{Anisotropy Analysis}

In this section, we discuss the anisotropy for the noncommutative Gaussian and Lorentzian geometries in order to examine the behavior of the anisotropic pressure. The role of anisotropy is significant in displaying the inner geometry of a relativistic wormhole configuration under the current conditions, and it is addressed as follows:

$$
\Delta=p_{t}-p_{r} .
$$

If $P_{r}>P_{t}$, the anisotropy is viewed as negative and is drawn inwards. However, if $P_{t}>P_{r}$, the anisotropy ends up being non-negative, which shows that the anisotropy is 
drawn outwards. The anisotropy for both the Gaussian and Lorentzian distributions with the linear model is calculated as

$$
\begin{aligned}
& \Delta=\frac{6 \sqrt{\pi}\left(\operatorname{Merf}\left(\frac{r}{2 \sqrt{\Theta}}\right)-4 \pi \alpha C_{1}\right)-\frac{M r e^{-\frac{r^{2}}{4 \Theta}\left(6 \Theta+r^{2}\right)}}{\Theta^{3 / 2}}}{16 \pi^{3 / 2} r^{3}}, \\
& \Delta=-\frac{6 \pi^{2} \alpha C_{2}\left(\Theta+r^{2}\right)^{2}+\sqrt{\Theta} M r\left(3 \Theta+5 r^{2}\right)-3 M\left(\Theta+r^{2}\right)^{2} \tan ^{-1}\left(\frac{r}{\sqrt{\Theta}}\right)}{4 \pi^{2} r^{3}\left(\Theta+r^{2}\right)^{2}} .
\end{aligned}
$$

Figures 23 and 24 represent the graphs of the anisotropy $P_{t}-P_{r}$ of the wormhole solutions for the linear and exponential models under the Gaussian and Lorentzian distributions. One can observe from the figures that $P_{t}-P_{r}>0$, i.e., the anisotropy is positive and, hence, directed outwards for both models. Hence, our wormhole configuration is stable.
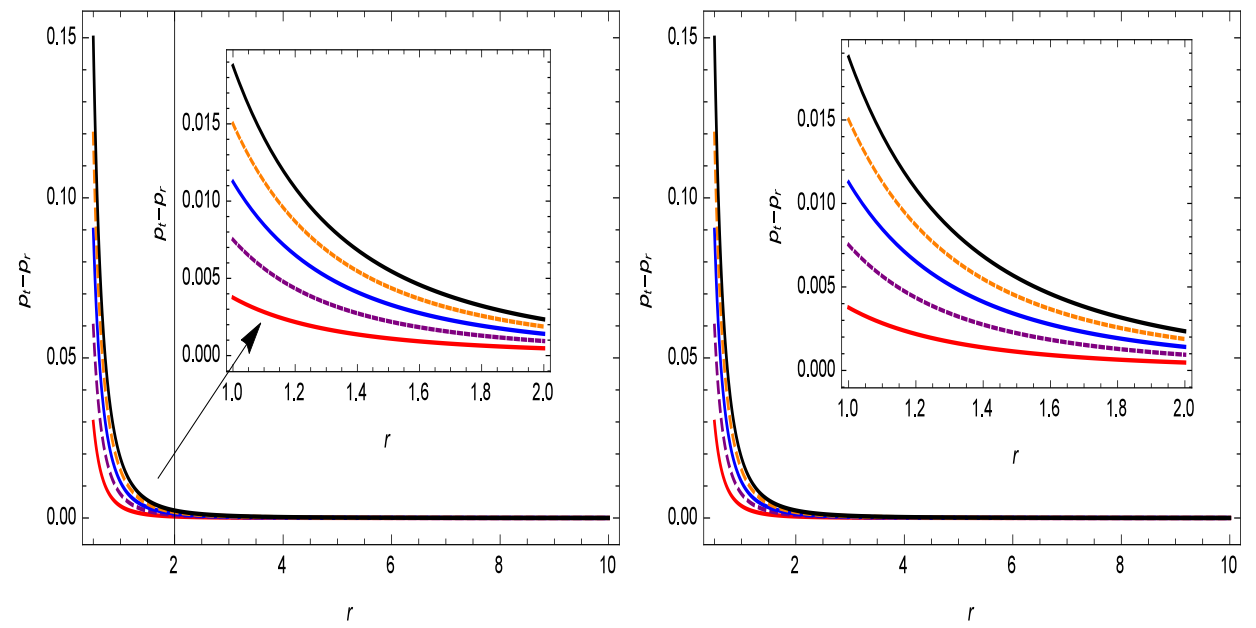

Figure 23. The graphical representation of $\Delta$ for the (left) Gaussian and (right) Lorentzian distributions for the linear model with $\alpha=-0.001(\star), \alpha=-0.002(\star), \alpha=-0.003(\star), \alpha=-0.004(\star)$, $\alpha=-0.005(\star), C_{1}=C_{2}=2.5, \Theta=0.5$, and $M=0.0007$.
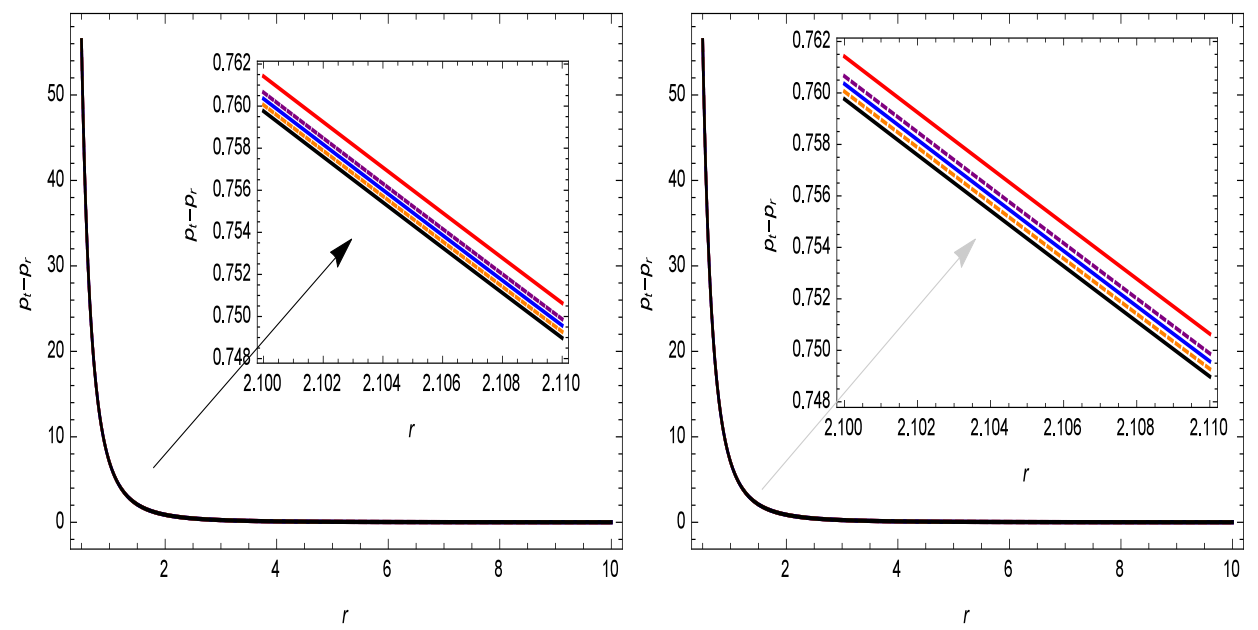

Figure 24. The graphical representation of $\Delta$ for the (left) Gaussian and (right) Lorentzian distributions for the exponential model with $\alpha=0.005(\star), \alpha=0.010(\star), \alpha=0.015(\star), \alpha=0.020(\star)$, $\alpha=0.025(\star), \Theta=0.5$, and $M=0.0007$. 


\section{Conclusions and Discussions}

Many researchers have an interest in the presence and creation of wormhole solutions with exotic matter in GR. The presence of exotic matter is quite possibly the main necessity for wormhole construction, as it is responsible for the violation of the null energy condition (NEC) and, hence, allows the wormhole's existence. This manuscript investigated wormhole solutions in symmetric teleparallel gravity with noncommutative Lorentzian and Gaussian backgrounds. We assumed two forms of $f(Q)$ gravity $-f(Q)=\alpha Q$ and $f(Q)=Q-\alpha\left(1-e^{-Q}\right)$, where $\alpha$ is the teleparallel gravitational term. The significant highlights of the current study are organized below.

First, we discussed the construction of a wormhole for the linear functional form of the $f(Q)$ model by considering the Gaussian and Lorentzian distributions, and we effectively examined the solutions for these distributions. To examine the physical behavior of our solutions, we plotted the shape function $b(r)$ against the radial coordinate $r$, which showed the increasing behavior of the shape function for both of the noncommutative distributions. We analyzed some fundamental characteristics of the shape function. In Figures 1-10, we clearly showed the behavior of the shape functions and energy conditions. These graphs show that the shape functions fulfill all of the requirements of the solution of the wormhole for both noncommutative sources. The energy density $\rho$ showed a clearly decreasing behavior for both noncommutative cases. The NEC was violated, as $\rho+P_{r}$ showed a negative behavior in both the Gaussian and Lorentzian cases. Thus, the presence of a wormhole in the noncommutative $f(Q)$ gravity is allowed because of the suitability of the solutions obtained.

Moving forward, we discussed the existence of a wormhole for the exponential model $f(Q)=Q-\alpha\left(1-e^{-Q}\right)$ by using Gaussian and Lorentzian sources. We obtained a complex differential equation for which it is practically difficult to obtain an analytic solution, so we settled it numerically. We can see from the left side of Figures 11 and 15 that the expanding behavior of the shape functions $b(r)$ was confirmed. Figures 11-20 show portions of the other essential conditions, such as the asymptotic flatness condition, flare-out condition, throat condition, energy density, and NEC. In this model, the energy density showed a clear downward behavior for both noncommutative sources. Moreover, the NEC was not satisfied, as the $\rho+P_{r}$ function showed a negative behavior for both noncommutative cases. The violation of the NEC in both cases is proof of the existence of exotic matter in the wormhole's throat. Thus, the conditions needed for the presence of wormholes are satisfied in these cases, and the wormhole's arrangements will keep on existing.

In addition, we considered the TOV equation to check how stable our wormhole solutions are. We investigated both $f(Q)$ models for their stability within sight of Lorentzian and Gaussian sources. When we surveyed the expressions of the anisotropic and hydrostatic forces, we investigated them using graphical means, as demonstrated in Figures 21 and 22. From these graphs, it is clear that these forces were similar in size, yet they were inverse in behavior, and thus canceled out each other's impact, implying that the wormhole that we calculated was balanced and stable. Furthermore, we performed an anisotropy analysis for the wormhole solutions that we obtained in the presence of the Gaussian and Lorentzian distributions. It is notable in Figures 23 and 24 that $P_{r} \neq P_{t}$ and $P_{t}>P_{r}$, indicating that $\Delta>0$. Thus, the anisotropy is positive and is coordinated outwards. Hence, our wormhole configuration is stable. This manuscript provides a detailed conversation on the presence of wormhole solutions in $f(Q)$ gravity by utilizing noncommutative geometry. Specifically, the acquired wormhole solutions are steady and adequate with the linear and exponential gravity models considered here. Hence, we can conclude that the results that we have calculated are stable and physically acceptable, as all of the calculated results satisfy all of the required properties. 
Author Contributions: Conceptualization, G.M.; methodology, G.M.; formal analysis, P.K.S.; resources, G.M.; data curation, Z.H., G.M.; investigation, Z.H.; writing-original draft, Z.H., G.M., P.K.S.; project administration, P.K.S. All authors made equal contributions to the work reported in this paper. All authors have read and agreed to the published version of the manuscript.

Funding: This research received no external funding.

Institutional Review Board Statement: Not applicable.

Informed Consent Statement: Not applicable.

Data Availability Statement: Not applicable.

Acknowledgments: Z.H. and P.K.S. acknowledge DST, New Delhi, India for providing facilities through the DST-FIST lab, Department of Mathematics, where a part of this work was completed. We are very grateful to the honorable referees and the editor for the illuminating suggestions that have significantly improved our work in terms of research quality and presentation.

Conflicts of Interest: The authors declare no conflict of interest.

\section{References}

1. Flamm, L. Beiträge zur Einsteinschen Gravitationstheorie. Phys. Z. 1916, 17, 448-454.

2. Misner, C.W.; Wheeler, J.A. Classical physics as geometry. Ann. Phys. 1957, 2, 525-603. [CrossRef]

3. Morris, M.S.; Thorne, K.S. Wormholes in spacetime and their use for interstellar travel: A tool for teaching general relativity. Am. J. Phys. 1988, 56, 395. [CrossRef]

4. Einstein, A.; Rosen, N. The Particle Problem in the General Theory of Relativity. Phys. Rev. 1935, 48, 73. [CrossRef]

5. Saiedi, H. Thermodynamics of Evolving Lorentzian Wormhole at Apparent Horizon in $f(R)$ theory of gravity. Mod. Phys. Lett. A 2012, 27, 1250220. [CrossRef]

6. Böhmer, C.G.; Harko, T.; Lobo, F.S.N. Wormhole geometries in modified teleparallel gravity and the energy conditions. Phys. Rev. D 2012, 85, 044033.

7. Lobo, F.S.N.; Oliveira, M.A. Wormhole geometries in $f(R)$ modified theories of gravity. Phys. Rev. D 2009, 80, 104012. [CrossRef]

8. Azizi, T. Wormhole Geometries in $f(R, T)$ Gravity. Int. J. Theor. Phys. 2013, 52, 3486-3493. [CrossRef]

9. DeBenedictis, A.; Horvat, D. On wormhole throats in $f(R)$ gravity theory. Gen. Relat. Gravit. 2012, 44, 2711-2744. [CrossRef]

10. Garcia, N.M.; Lobo, F.S.N. Exact solutions of Brane-Dicke wormholes in the presence of matter. Mod. Phys. Lett. A 2011, 40, 3067. [CrossRef]

11. Jamil, M.; Momeni, D.; Myrzakulov, R. Wormholes in a viable $f(T)$ gravity. Eur. Phys. J. C 2013, 73, 2267. [CrossRef]

12. Jimenez, J.B.; Heisenberg, L.; Koivisto, T. Coincident general relativity. Phys. Rev. D 2018, 98, 044048. [CrossRef]

13. Harko, T.; Koivisto, T.S.; Lobo, F.S.N.; Olmo, G.J.; Garcia, D.R. Coupling matter in modified Q gravity. Phys. Rev. D 2018, 98, 084043. [CrossRef]

14. Mandal, S.; Sahoo, P.K.; Santos, J.R.L. Energy conditions in $f(Q)$ gravity. Phys. Rev. D 2020, 102, 024057. [CrossRef]

15. Barros, B.J.; Barreiro, T.; Koivisto, T.; Nunes, N.J. Testing $F(Q)$ gravity with redshift space distortions. Phys. Dark Universe 2020, 30, 100616. [CrossRef]

16. Solanki, R.; Pacif, S.K.J.; Parida, A.; Sahoo, P.K. Cosmic acceleration with bulk viscosity in modified $f(Q)$ gravity. Phys. Dark. Universe 2021, 32, 100820. [CrossRef]

17. Mandal, S.; Wang, D.; Sahoo, P.K. Cosmography in $f(Q)$ gravity. Phys. Rev. D 2020, 102, 124029. [CrossRef]

18. Lazkoz, R.; Lobo, F.S.N.; Banos, M.O.; Salzano, V. Observational constraints of $f(Q)$ gravity. Phys. Rev. D 2019, $100,104027$. [CrossRef]

19. Jimenez, J.B.; Heisenberg, L.; Koivisto, T.; Pekar, S. Cosmology in $f(Q)$ geometry. Phys. Rev. D 2020, 101, 103507. [CrossRef]

20. Hassan, Z.; Mandal, S.; Sahoo, P.K. Traversable wormhole geometries in $f(Q)$ gravity. Fortschr. Phys. 2021, 69, 2100023. [CrossRef]

21. Nicolini, P.; Smailagic, A.; Spalluci, E. Noncommutative geometry inspired Schwarzschild black hole. Phys. Lett. B 2006, 632, 547-551. [CrossRef]

22. Schneider, M.; DeBenedictis, A. Noncommutative black holes of various genera in the connection formalism. Phys. Rev. D 2020, 102, 024030. [CrossRef]

23. Seiberg, N.; Witten, E. String theory and noncommutative geometry. J. High Energy Phys. 1999, 9, 32. [CrossRef]

24. Gruppuso, A. Newton's law in an effective non-commutative space-time. J. Phys. A Math. Gen. 2005, 38, 2039. [CrossRef]

25. Smailagic, A.; Spallucci, E. Feynman path integral on the non-commutative plane. J. Phys. A Math. Gen. 2003, 36, L467. [CrossRef]

26. Rahaman, F.; Islam, S.; Kuhfittig, P.K.F.; Ray, S. Searching for higher-dimensional wormholes with noncommutative geometry. Phys. Rev. D 2012, 86, 106010. [CrossRef]

27. Sushkov, S. Wormholes supported by a phantom energy. Phys. Rev. D 2005, 71, 043520. [CrossRef]

28. Nicolini, P.; Spalluci, E. Noncommutative geometry-inspired dirty black holes. Class. Quant. Grav. 2010, 27, 015010. [CrossRef]

29. Abreu, E.M.C.; Sasaki, N. Noncommutative wormholes and the energy conditions. arXiv 2012, arXiv:1207.7130. 
30. Jamil, M.; Rahaman, F.; Myrzakulov, R.; Kuhfittig, P.K.F.; Ahmed, N.; Mondal, U.F. Nonommutative wormholes in $f(R)$ gravity. arXiv 2013, arXiv:1304.2240.

31. Raychaudhuri, A. Relativistic Cosmology. I. Phys. Rev. D 1955, 98, 1123. [CrossRef]

32. Nojiri, S.; Odintsov, S.D. Introduction to modified gravity and gravitational alternative for dark energy. Int. J. Geom. Methods Mod. Phys. 2007, 4, 115-145. [CrossRef]

33. Ehlers, J.A.K. Raychaudhuri and his equation. Int. J. Mod. Phys. D 2006, 15, 1573-1580. [CrossRef]

34. Elizalde, E.; Khurshudyan, M. Wormhole models in $f(R, T)$ gravity. Int. J. Mod. Phys. D 2019, 28, 1950172. [CrossRef]

35. Elizalde, E.; Nojiri, S.; Odintsov, S.D.; Sebastiani, L.; Zerbini, S. Nonsingular exponential gravity: A simple theory for early- and late-time accelerated expansion. Phys. Rev. D 2011, 83, 086006. [CrossRef]

36. Oppenheimer, J.R.; Volkoff, G.M. On Massive Neutron Cores. Phys. Rev. 1939, 55, 374. [CrossRef]

37. Kuhfittig, P.K.F. A note on the stability of Morris-Thorne wormholes. arXiv 2020, arXiv:2009.11179v1.

38. Gorini, V.; Moschella, U.; Kamenshchik, A.Y.; Pasquier, V.; Starobinsky, A.A. Tolman-Oppenheimer-Volkoff equations in the presence of the Chaplygin gas: Stars and wormholelike solutions. Phys. Rev. D 2008, 78, 064064. [CrossRef] 